This item was submitted to Loughborough's Research Repository by the author.

Items in Figshare are protected by copyright, with all rights reserved, unless otherwise indicated.

\title{
PIV study of the flow field generated by a sawtooth impeller
}

PLEASE CITE THE PUBLISHED VERSION

http://dx.doi.org/10.1016/j.ces.2011.07.046

PUBLISHER

(C) Elsevier

VERSION

AM (Accepted Manuscript)

LICENCE

CC BY-NC-ND 4.0

REPOSITORY RECORD

Unadkat, Heema, Chris D. Rielly, and Zoltan K. Nagy. 2013. "PIV Study of the Flow Field Generated by a Sawtooth Impeller". figshare. https://hdl.handle.net/2134/11471. 
This item was submitted to Loughborough's Institutional Repository (https://dspace.lboro.ac.uk/) by the author and is made available under the following Creative Commons Licence conditions.

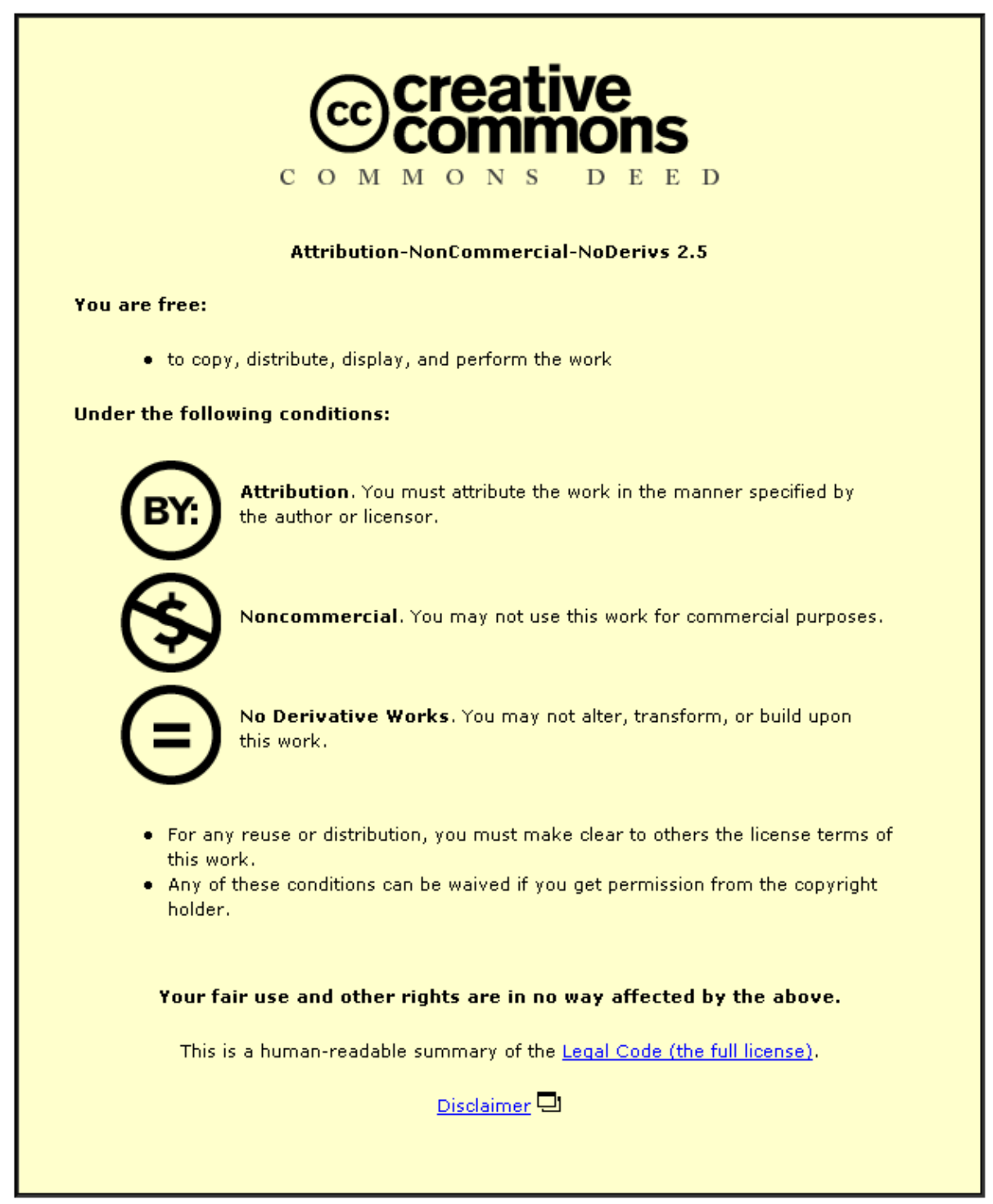

For the full text of this licence, please go to: http://creativecommons.org/licenses/by-nc-nd/2.5/ 


\title{
PIV study of the flow field generated by a sawtooth impeller
}

\author{
H.Unadkat, C.D Rielly and Z.K.Nagy \\ Department of Chemical Engineering \\ Loughborough University, Loughborough, Leics LE11 3TU, UK
}

\begin{abstract}
Stereoscopic and high-speed particle image velocimetry (PIV) techniques have been employed to study the flow field induced by a sawtooth (EkatoMizer) impeller, operated in the fully turbulent flow regime at an impeller speed of $1500 \mathrm{rpm}$. Ensemble-averaged mean flow fields and turbulence quantities were calculated for a region close to the impeller blades. The flow was found to be anisotropic near the impeller and exhibited return-to-isotropy behaviour further away from it. Macroinstabilities were found to have a high probability of occurrence in the discharge stream. All three velocity components from the stereo-PIV measurements were used to estimate the dissipation rate, by adopting a large eddy simulation (LES) analogy. Spurious vectors distorting the dissipation rate calculation were identified, and various standard deviation filters were applied for vector validation. By evaluating the filtered dissipation rate profiles against the multi-fractal intermittency model of Meneveau and Sreenivasan (1991), the global standard deviation filter was found to be the most suitable type. The ratio of the maximum to the mean dissipation rate for the EkatoMizer discharge stream was found to be similar to that reported for Rushton disk turbine and pitched-blade turbine impellers in the literature, raising questions about the reported high-shear advantage of sawtooth impellers.
\end{abstract}

Keywords: Particle image velocimetry; Stirred vessels; Energy dissipation; Turbulence, Intermittency, Multifractal Model 


\section{Introduction}

Flows in stirred tanks are often complex and multi-dimensional and have a very significant effect on the mixing processes. Mechanically agitated tanks are used for a variety of industrial operations, such as blending of miscible or immiscible solvents, dispersion of gases or solids in liquids, chemical reactions and for processes ranging from polymer production to fermentations (Kresta and Wood, 1993). The complexity of stirred flows in chemical reactors has motivated a number of studies to investigate the mean flow patterns and turbulence properties of the flow fields generated by various types of impellers.

The choice of an impeller is largely dependent on the mixing requirements; the intensity of agitation may be characterised by the relative distribution of the mean and turbulent kinetic energy (TKE), $k$, and more importantly the TKE dissipation rate, $\varepsilon$. The demand for improved impeller designs is driven by the need to enhance the quality and capacity of existing processes, or to satisfy new technologies. One such example is nano-particle technology, which is a developing field of research at the forefront of current investigations and involves the synthesis and controlled dispersion of particles on a scale considerably smaller than $1 \mu \mathrm{m}$. The sawtooth impeller is claimed to be a high shear mixing device, which may be used to deagglomerate nano-particle dispersions. Compared to Rushton disk turbines (RDTs) and pitched-blade turbines (PBTs), which have received a lot of attention over the last three decades (e.g. van't Riet and Smith, 1975; Wu and Patterson, 1989; Lee and Yianneskis, 1998; Yianneskis and Whitelaw, 1993; Khan et al., 2004; Gabriele et al., 2009), the hydrodynamics of sawtooth impellers are relatively unknown, and have not been widely investigated. Consequently, a study has been carried out to investigate the flow field and turbulence properties generated by an EkatoMizer, which is a type of sawtooth impeller. Recently, studies have been carried out to investigate the dispersion of nano-particle agglomerates by a sawtooth impeller (Xie et al., 2007; Xie et al., 2008), but the present contribution focuses on the local hydrodynamics of the flow close to the rotating blades.

In the current work, low speed $(15 \mathrm{~Hz})$ stereoscopic particle image velocimetry (stereo-PIV) has been employed to obtain three-dimensional flows close to the sawtooth blades of an EkatoMizer impeller. The PIV measurement system employs 
a two-camera set-up, arranged in a Scheimpflug configuration (Prasad, 2000). Highspeed $(5 \mathrm{kHz})$, two-dimensional PIV experiments were also carried out to obtain better time-resolved velocity fields. Mean flow properties of the impeller will be presented, including an analysis of flow anisotropy and macro-instabilities.

The local dissipation rate, $\varepsilon$, is of particular interest, as it is a key variable related to the breakage of nano-particle clusters (Xie et al., 2008). The calculation of $\varepsilon$ has been the subject of many previous works (e.g. Kresta and Wood, 1993; Wu and Patterson, 1989; Zhou and Kresta, 1996; Baldi and Yianneskis, 2003; Ducci and Yianneskis, 2005; Sharp and Adrian, 2001; Khan, 2005, Kilander and Rasmuson, 2005; Sheng et al., 2000); a comparison between the different methods has not been provided in this paper (but may be found in the work of Unadkat (2010)). It is now well known that the LES analogy provides the best approximation (Khan, 2005; Gabriele et al., 2009), and hence this method has been employed here to estimate the dissipation rate.

This paper also addresses the issue of spurious vectors in raw PIV measurements, which lead to the calculation of erroneous velocity gradients and have a compounding effect on the dissipation rate estimate in the LES analogy method. A technique has been developed to distinguish between spurious results and genuine turbulence intermittency, by comparing the generalized dimensions of the time-history dissipation rate profiles with the multi-fractal model of Meneveau and Sreenivasan (1991). High-speed PIV data were crucial for this analysis. The proposed method was also found to be useful for determining an appropriate standard deviation filter used for vector validation. The final section of this paper considers the ratio of the maximum and mean dissipation rate for the EkatoMizer, and provides a comparison with other impellers on this basis.

\section{Experimental Setup}

Two independent experiments were carried out, using stereo- and high-speed PIV. Both techniques are described in this section.

\subsection{Stereoscopic PIV}

A standard cylindrical Perspex tank with a torispherical base and inner diameter of $T=305 \mathrm{~mm}$ was employed for these experiments. The sawtooth impeller 
(EkatoMizer) of diameter $D=T / 3.21$ was operated at a clearance of $C=T / 3$. The tank was equipped with four wall baffles, each of width $B=0.1 T$, which were located at $90^{\circ}$ intervals around the tank wall. The fluid (deionised water) was filled to a height $H=T$, and the liquid surface was open to the atmosphere. As shown in Fig. 1, the cylindrical tank was placed inside a larger pentagonal Perspex tank filled with the same working fluid, to eliminate refraction of the laser light sheet at the curved surface. The front two faces of the tank intersected at an angle of $120^{\circ}$.

The stereo-PIV setup is illustrated in Fig. 1. The recording device consisted of two CCD cameras from TSI Insight, with a resolution of $1000 \times 1016$ pixels (TSI PIVCAM 10-30 model 630046) which were positioned perpendicular to the front two faces of the outer tank. In this arrangement, the lens axes of both cameras intersected at $60^{\circ}$ on the object plane, forming an off-set half angle of $30^{\circ}$. This arrangement, known as the Scheimpflug configuration, provides an accurate velocity measurement for non-uniform magnification. The system was equipped with a double-pulsed New-Wave Nd:YAG laser of $532 \mathrm{~nm}$ wavelength. The laser beam was passed through a cylindrical lens, which generated a light sheet of approximately $1 \mathrm{~mm}$ thickness. The laser had a maximum repetition rate of $15 \mathrm{~Hz}$ (for double pulses) with a pulse width of 5 to $7 \mathrm{~ns}$, and a maximum pulse energy of $50 \mathrm{~mJ}$. To minimise light reflections off the impeller surface, the disc and shaft were painted matt black.

The impeller was operated at 1500 rpm, corresponding to a Reynolds number of $R e=\rho N D^{2} / \mu=2.3 \times 10^{5}$, thus satisfying operation within the turbulent flow regime $\left(\operatorname{Re}>4 \times 10^{3}\right)$. The fluid was seeded with $10 \mu \mathrm{m}$ hollow glass spheres. Three positions near the impeller were investigated, as depicted in Fig. 2 (Positions 1, 2 and 3 corresponding to the impeller region, discharge stream and in front on the blade, respectively). In each experiment, 500 image pairs were acquired for each camera. The EkatoMizer had 36 upper teeth and 36 lower teeth, spaced very closely together (see Fig. 3). Since it was not possible to determine accurately the positions of the individual teeth, measurements were ensemble-averaged, i.e. taken around $360^{\circ}$ of the impeller.

The most crucial stage for any stereo-PIV experiment is the calibration, as this determines the accuracy of the out-of-plane velocity component, accounts for the 
local magnifications in the three directions, and corrects the centre of the field of view for the two cameras when not precisely aligned. It is also required to correct the image perspective distortions inherent with the Scheimpflug configuration. For the calibration, a target grid containing calibration markers (black dots) against a white background, arranged on a Cartesian grid, was placed in the object plane to coincide exactly with the light sheet. The grid was mounted on a micrometer for fine tuning. Calibration was achieved by acquiring images of the grid on each camera, as it was traversed through the laser light sheet in five small increments $(0.25 \mathrm{~mm})$ in the outof-plane $z$-direction. Using knowledge of the true positions of the calibration points, the PIVCalib program was able to map the object plane $(x, y, z)$ to the image plane $(X, Y, Z)$. Cross-correlation of the images was carried out using TSI Insight software. For all images, the interrogation area $(I A)$ was $32 \times 32$ pixels, with $50 \%$ overlap. The PIV resolution was $1 \mathrm{~mm}$ (corresponding to the length of one side of the $I A$ ) and the field of view was $30.5 \times 31.5 \mathrm{~mm}$. A range filter was applied to the raw data to remove obvious spurious vectors whose velocities exceeded $1.5 \times V_{\text {tip }}$ (where $V_{\text {tip }}=\pi N D$ is the tip velocity). The resultant holes were filled via linear interpolation of the surrounding vectors.

\subsection{High-speed PIV}

High-speed PIV experiments were also carried out. The components were the same as those used for the stereo-PIV experiments, and hence only differences in the measurement techniques will be described. The images were acquired using a single camera (analogous to standard 2-D PIV), and two in-plane velocity components were obtained (in the axial and radial directions). The outer pentagonal tank was rotated $90^{\circ}$ clockwise such that the camera was aligned perpendicular to a flat surface. The laser used was a New-Wave Nd:YLF Pegasus with a wavelength of $527 \mathrm{~nm}$. For camera calibration, the aforementioned target grid was placed in the centre of the light sheet, and a single image was captured, after which the pixel-to$\mathrm{mm}$ conversion was deduced manually. The image acquisition rate was $5 \mathrm{kHz}$, and a total of 1000 image pairs were captured in each experiment. For the crosscorrelation, $I A$ sizes of $16 \times 16$ and $32 \times 32$ were employed for the first and second images (in each pair) respectively, to improve the correlation around the edges of the $I A$ in the first image. No IA overlap was introduced. The PIV resolution varied 
between 0.6-0.7 $\mathrm{mm}$ in the various trials (corresponding to the size of an $I A$ in the first image) and the field of view was approximately $43 \times 43 \mathrm{~mm}$. As in the stereoPIV measurements, a range filter was applied to eliminate vectors whose velocities exceeded $1.5 \times V_{\text {tip }}$.

\section{Mean flow properties}

This section describes the mean flow field generated by the EkatoMizer, obtained from the stereo-PIV measurements at Position 1 and at $1500 \mathrm{rpm}\left(\operatorname{Re}=2.3 \times 10^{5}\right)$. For presentation purposes, the data has been plotted on the $r-z$ (radial-axial) plane according to polar coordinates. In the figures, the radial mean velocities are denoted by use of angled brackets $\langle u>$ etc. and all velocities have been normalised by the tip speed $V_{\text {tip }}=\pi N D=7.46 \mathrm{~m} / \mathrm{s}$.

\subsection{Mean velocity}

The mean axial and radial velocity vector components are presented in Fig. 4, which enables visualisation of the discharge stream. Despite the fact that the sawteeth are aligned in the tangential direction, there is a strong radial discharge flow, with axial suction intakes above and below the EkatoMizer disk. The maximum radial velocity $\left(\sim 0.12 V_{\text {tip }}\right)$ is found at the blade edge on the centreline, whereas the mean axial flow exhibits a maximum $\left(\sim 0.03 V_{\text {tip }}\right)$ above and below the impeller. The two velocity components appear to be almost spatially segregated, indicating that there is no mixed flow. However, visual observations of instantaneous velocity fields (as in Fig. 5) elucidated vortex structures appearing randomly within the discharge stream. Frequently, two vortices formed simultaneously on either side of the impeller centreline plane; one was located in the upper half of the discharge stream whilst the second remained in the lower half. The vortices had a maximum displacement of around $7 \times 10^{-4} \mathrm{~m}$ between image pairs, and persisted for approximately $2 \mathrm{~ms}$ before disappearing and instantly reforming at other positions within the discharge stream. This time scale compares with the blade passage frequency of about $0.5 \mathrm{~ms}$ for top and bottom teeth, or $1.0 \mathrm{~ms}$ for the top teeth alone. Although the vortices were noticed in the stereo-PIV vector plots, their displacement and life-time was obtained from high-speed PIV data, as the time separation between image pairs in the stereoPIV experiments was too long to enable their tracking. These turbulent structures are 
effectively smoothed out during the averaging process; although the mean velocity fields enable analysis of the dominant flow patterns, they provide no information of the unsteady flow structures.

Finally, the mean tangential velocity, obtained in the field of view corresponding to Position 1 is presented in Fig. 6 (a). The range of tangential velocities marginally exceeds that of the radial velocities, reaching a maximum of $\sim 0.14 V_{t i p}$ (negative velocities indicate a flow direction into the page). Although the maximum tangential velocity exceeds the maximum radial velocity, it is not as evenly distributed across the field of view. The maximum tangential velocity is found at the blade edge $(r / T=0.156)$, similar to the radial velocity measurements, but the large values are confined very much to the impeller discharge region. Thus the strong tangential components are directly attributable to the impeller motion itself, and result from the flow being dragged behind the impeller blade. The mean flow fields obtained at Position 2 (Fig. 6 (b)) illustrated that the relatively strong tangential flow decayed more rapidly than the radial flow further away from the blade. Consequently, the radial mean velocity component persists and is the predominant flow direction in the discharge stream.

\section{2 rms velocities and TKE}

Each rms velocity component is defined as

$$
\tilde{u}_{i}=\sqrt{\overline{\overline{u_{i}^{\prime 2}}}}=\sqrt{\overline{\left(u_{i}-\overline{u_{i}}\right)^{2}}}
$$

where $u_{i}^{\prime}$ is the fluctuating velocity in the $i$ direction and $\bar{u}_{i}$ is the time-averaged velocity. Normalized axial and radial rms velocities in the impeller region (Position 1) at $1500 \mathrm{rpm}$ are shown in Fig. 7 (a) and (b) respectively. Their distributions are similar, although they differ slightly in magnitude. Their maximum values are $0.05 V_{t i p}$ and $0.06 V_{\text {tip }}$ respectively; a difference of $20 \%$. Stereo-PIV also enables the measurement of the tangential rms velocity, as displayed in Fig. 7 (c). The tangential rms velocity is larger than the axial or radial components, with a maximum of $0.1 V_{\text {tip }}$. For pseudo-isotropic flows $\tilde{u}^{2} \approx \tilde{v}^{2} \approx \tilde{w}^{2}$ and under this assumption, the tangential rms velocity may be approximated as $\tilde{w}^{2}=\left(\tilde{u}^{2}+\tilde{v}^{2}\right) / 2$. If the assumption was valid for the 
EkatoMizer flow, the maximum tangential rms velocity would be $\sim 0.055 V_{t i p}$. The true value clearly indicates flow anisotropy, and suggests that the isotropic assumption underestimates the turbulence level. Consequently, although standard 2-D PIV may provide a reliable estimate of the measurable components, it is inadequate in predicting the turbulence in the out-of-plane direction using an isotropic assumption.

The turbulent kinetic energy (TKE) $k$ was calculated from its full definition, using all three velocity components:

$$
k=\frac{1}{2}\left(\overline{u^{12}}+\overline{v^{12}}+\overline{w^{12}}\right)
$$

The result is displayed in Fig. 8, where the TKE reaches a maximum of $0.008 V_{t i p}^{2}$ at the blade edge, consistent with the axial and radial rms velocity regions of maxima. Note that a pseudo-isotropic assumption made in the calculation of the TKE (using only the axial and radial rms velocities) provided a lower estimate of the TKE near the blade edge $\left(0.005 V_{\text {tip }}^{2}\right)$. As expected, this is because the isotropic assumption underestimates the tangential rms velocity (by approximately $45 \%$ in the present case).

\subsection{Flow anisotropy}

Flow anisotropy was determined via the anisotropy tensor $a_{i j}$ which is characterized using the Reynolds stresses (Pope, 2000):

$$
a_{i j}=\overline{u_{i}^{\prime} u_{j}^{\prime}}-\frac{2}{3} k \delta_{i j}
$$

Above $\delta_{i j}$ is the Kronecker delta, which is 1 if $i=j$ and 0 if $i \neq j$. The normalized anisotropy tensor is defined as:

$b_{i j}=\frac{a_{i j}}{2 k}=\frac{\overline{u_{i}^{\prime} u_{j}^{\prime}}}{\overline{u_{k}^{\prime} u_{k}^{\prime}}}-\frac{1}{3} \delta_{i j}$

The normalized anisotropy tensor has zero trace $\left(b_{j j}=0\right)$, meaning that the first invariant is zero by definition. The second and third invariants are given below respectively. 
$b_{i i}^{2}=6 \eta^{2}=b_{i j} b_{j i}$

$b_{i i}^{3}=6 \xi^{3}=b_{i j} b_{j k} b_{k i}$

From the above equations, anisotropy may then be characterized by the two variables denoted $\xi$ and $\eta$. For all points in the flow field, $\xi$ and $\eta$ may be determined from the Reynolds stresses and plotted as points on the $\xi-\eta$ plane. There exist particular states of the Reynolds stress tensor that correspond to specific points and curves in this plane, which in turn describe the state of turbulence.

When axisymmetric turbulence is present, the kinetic energy in two orthogonal directions is equal. Subsequently, the anisotropy tensor may be written as:

$$
b_{i j}=\left(\begin{array}{ccc}
-\alpha & 0 & 0 \\
0 & \alpha / 2 & 0 \\
0 & 0 & \alpha / 2
\end{array}\right)
$$

The parameter $\alpha$ describes the energy content in the three coordinate directions. The energy contained in the first direction is $\left(\frac{1}{3}-\frac{1}{2} \alpha\right) k$ (Derksen et al., 1999). In this case, the invariants maybe written as $b_{i i}^{2}=\frac{3}{2} \alpha^{2}$ and $b_{i i}^{3}=-\frac{3}{4} \alpha^{3}$. It follows that when $\alpha<0, b_{i i}^{3}=6\left(\frac{b_{i i}^{2}}{6}\right)^{3 / 2}$. For this case, the energy contained in the direction of symmetry is greater than $k / 3$. Conversely, when $\alpha>0$, then $b_{i i}^{3}=-6\left(\frac{b_{i i}^{2}}{6}\right)^{3 / 2}$ and the energy in the direction of symmetry is less than $k / 3$. The equivalent expressions for these conditions in terms of the variables $\xi$ and $\eta$ are $\eta=\xi$ and $\eta=-\xi$ respectively, for an anisotropic flow with an axisymmetric state of turbulence. These curves create a boundary for the anisotropic conditions, and represent the Lumley triangle (Lumley, 1978). The bottom corner of the triangle where $\eta=\xi=0$ corresponds to an isotropic state of turbulence. This suggests that for three-dimensional isotropic flows, all invariants of the Reynolds anisotropy tensor are essentially zero.

The values of $\xi$ and $\eta$ for the flow field measured at Position 1, $1500 \mathrm{rpm}$, are shown in Fig. 9. The coloured points correspond to different sections of the flow 
field. In order of increasing distance from the impeller, the colours are blue, red, yellow, dark green and bright green. All points within the Lumley triangle correspond to physically realizable Reynolds stresses, whereas outliers correspond to nonrealizable Reynolds stress. As may be observed, all of the measurement points are found near the right hand side of the Lumley triangle, within the $\eta=\xi$ boundary. The first conclusion that may be obtained is that some points within the flow field are highly anisotropic. Secondly the state of turbulence may be categorized as axisymmetric, with positive $\xi$. This further suggests that the direction of symmetry has the highest energy content. An analysis of the $b_{i j}$ components indicated that the tangential plane was the direction of symmetry for the flow generated by the EkatoMizer. No points within the flow field satisfy the condition of isotropy and hence the bottom corner of the Lumley triangle is empty.

The blue points, corresponding to the immediate vicinity of the impeller, display the highest state of anisotropy, and fall closest to the region $\eta=\xi=1 / 3$, relative to all other points. Conversely, the bright green points located furthest away from the impeller show the highest degree of isotropy, and are located close to $\eta=\xi=0$ (although they are not exactly isotropic). The remaining red, yellow and dark green points fall between these two limits, and in that order show return-toisotropy behaviour. For a similar analysis carried out at Position 2 (further into the discharge stream, but not shown here), all coloured points were closer to isotropy, and occupied a larger area in the bottom corner of the Lumley triangle. Hence the turbulence becomes more isotropic with increasing distance from the sawtooth impeller.

\subsection{Macro-instabilities}

Macro-instabilities (MI) are large-scale, low-frequency flow instabilities whose time and length scales are greater than those associated with the impeller blade rotation and small scale turbulence eddies. Earlier findings suggest that $\mathrm{MI}$ are responsible for slowly changing the flow pattern in stirred vessels about its mean. The effects are manifested in quantities such as the local heat flux (Haam et al., 1992) or mass fluxes in multiphase systems (Bittorf and Kresta, 1999). Consequently, an understanding of the degree, nature and mechanisms of $\mathrm{Ml}$ formation are of great 
importance in the mixing field. It is possible that the vortices found in the instantaneous PIV results (Fig. 5) correspond to low-frequency phenomena.

Information from the high-speed PIV experiments was used to perform a spectral analysis of the velocity time-series. In order to take full advantage of the spatial information carried in PIV data, the distribution of MI intensity across the field of view was obtained, in a manner analogous to Fan et al. (2004). The power spectral density (PSD) obtained at a discrete point in the flow field (for $N=1500 \mathrm{rpm}$ ) is shown in Fig. 10, which illustrates the variability in MI peaks at frequencies much lower than the blade passage (e.g. $900 \mathrm{~Hz}$ for passage of the upper teeth). In most points of the flow field, dominant peaks in the low frequency region were not identifiable; instead two or three significant peaks occurred simultaneously. In locations where a single peak could be identified, the frequency was not always the same. Consequently, an explicit value of the dominant Ml frequency could not be determined. Similar types of difficulties in determining the dominant MI peak have been noted in the works of Montes et al. (1997), Hasal et al. (2000), Nikiforaki et al. (2003) and Khan (2005). However, this was not crucial in the analysis. When dealing with the probability of $\mathrm{Ml}$ occurrence, the $\mathrm{Ml}$ frequency range is a more important factor. The frequency $(t)$ of the $\mathrm{MI}$ in the current study was found to be within the range $0-200 \mathrm{~Hz}$.

The $z$ (out of plane) component of vorticity was calculated for every point in the velocity field (using central difference approximations to the derivatives), and for each instantaneous PIV measurement in the series. Subsequently, time series of the vorticities were obtained at every point in the flow field. The signals were processed by calculating the PSD $h(f)$ from a periodogram estimate. The normalized PSD $F(f)$ was obtained by dividing the non-normalized PSD by the variance of the vorticity time series. Finally, the spectral distribution function $P(f)$ was calculated to quantify the Ml. According to Fan et al. (2004) $P(f)$ may be regarded as the proportion of the total power contributed by the components with frequencies within the range 0 and $f$. In this case $f$ is $200 \mathrm{~Hz}$.

$$
F(f)=\frac{h(f)}{\sigma^{2}}
$$


$P(f)=\int_{0}^{f} F(\theta) D \theta$

Fan et al. (2004) also suggested that from a mathematical perspective, $P(f)$ is equivalent to a probability distribution function. For instance, $P(f)=0.5$ means that the $\mathrm{Ml}$ dominates $50 \%$ of the total measurement period, at that measurement location. $P(f)$ may also be interpreted as the $\mathrm{Ml}$ intensity. The 2-D distribution of the probability of $\mathrm{MI}$ occurrence in the measurement region is depicted in Fig. 11. It may be observed that $\mathrm{Ml}$ intensity is significantly higher in the discharge stream compared to the surrounding flow, with a magnitude of approximately 0.8 . In other words, Ml contributes to around $80 \%$ of the total power within that region. The result suggests that the vortical structures observed in Fig. 5 may be low-frequency $\mathrm{Ml}$ detected in the PSD distribution, since the region of high intensity coincides with their location.

The above description of $\mathrm{Ml}$ may be in contrast with the type of $\mathrm{Ml}$ phenomena reported in literature for RDT and PBT impellers (Yianneskis et al., 1987; Haam et al., 1992; Guillard et al., 2000; Nikiforaki et al., 2003). A common factor amongst the aforementioned studies is that Mls persist close to the liquid surface, circulate around the shaft or form behind the baffles, whereas in the present case, the Mls have a relatively larger probability of occurrence in the discharge stream. Secondly, the Ml frequencies (both absolute $f$ and normalized $f^{\prime}=f / N$ ) recorded previously for RDT and PBT impellers $\left(0.017<f^{\prime}<0.036\right.$ in the listed studies) are approximately two orders of magnitude smaller than those found in the present case (reaching $f^{\prime}=8$ ). From the instantaneous vector plots of the highspeed PIV measurements, it was found that vortices persisted for approximately 2 ms before disappearing, and instantly reforming at other positions in the discharge stream. This type of behaviour does not conform to the traditional view of $\mathrm{Ml}$, which endure for longer periods (e.g. $10 \mathrm{~s}$ identified by Yianneskis et al. (1987) for an RDT). The only firm conclusion that can be drawn thus far is that low-frequency phenomena (between $0-200 \mathrm{~Hz}$, and hence lower relative to the tooth passage frequency of $900 \mathrm{~Hz}$ ) have been detected for the EkatoMizer. The results of the current study create the possibility of characterising another form of low-frequency 
phenomena, which appropriately describes the flow behaviour of the sawtooth impeller.

\section{Dissipation rate}

A direct determination of the dissipation rate requires spatial gradients of the turbulent velocity field to be resolved down to the Kolmogorov scale for an accurate estimate (Ducci and Yianneskis, 2005), but this is not within the capability of PIV experiments in a stirred turbulent flow. Saarenrinne et al. (2001) suggested that a spatial resolution of $2 \eta$ is required to estimate approximately $90 \%$ of the true dissipation rate. The mean Kolmogorov length scale in the bulk flow (from the mean dissipation rate of $0.6 \mathrm{~W} \mathrm{~kg}^{-1}$ at $N=25 \mathrm{rps}$ and $P_{o}=0.11$ ) was estimated to be around $36 \mu \mathrm{m}$. It follows that the PIV resolution of $28 \eta$ (at the $1 \mathrm{~mm}$ filtering scale) is insufficient to determine the dissipation rate directly from its fundamental definition. Consequently, the large eddy simulation (LES) analogy of Sheng et al. (2000) was employed for the calculation, which was assumed to be the best method compared to dimensional analyses (e.g. $\varepsilon=A k^{3 / 2} /\left(3 \Lambda^{2}\right)^{1 / 2}$, where $\Lambda$ is an integral length scale and $A$ is a constant of $\mathrm{O}(1))$ and the direct estimate. Comparisons between the various calculation methods of $\varepsilon$ have been presented by Unadkat (2010), and are not the focus of the current paper. The conclusion that the large eddy analogy of Sheng et al. (2000) is a useful method to calculate $\varepsilon$, is in agreement with the works of Khan (2005) and Gabriele et al. (2009).

In the LES analogy, the resolved scale velocity field is that measured using PIV, and the unresolved scale effects are included using a sub-grid scale (SGS) model. The $I A$ size is naturally the spatial filter. The turbulence dissipation rate $\varepsilon$ can be approximated by computing the Reynolds averaged SGS dissipation rate $\overline{\varepsilon_{S G S}}$ at the PIV resolution:

$$
\varepsilon \approx \overline{\varepsilon_{S G S}}=-2 \overline{\tau_{i j} s_{i j}}
$$

The Reynolds average on the right hand side of Eq. (10) is a time-average over sequential full-field PIV data sets and $s_{i j}$ is the resolved scale strain rate tensor calculated from gradients of the instantaneous velocity fields: 
$s_{i j}=\frac{1}{2}\left(\frac{\partial u_{j}}{\partial x_{i}}+\frac{\partial u_{i}}{\partial x_{j}}\right)$

In Eq. (10), $\tau_{i j}$ is the SGS stress tensor, which must be modelled by a SGS turbulence model. Various SGS models have been suggested, such as the Smagorinsky model (Smagorinsky, 1963), similarity model (Lui et al., 1994) and gradient model (Clark et al., 1979). Sheng et al. (2000) found that both the Smagorinsky and similarity models yielded consistent results for the dissipation rate. Hence, the Smagorinsky model of Eq.(11) was employed in this study.

$\tau_{i j}=-C_{s}^{2} \Delta^{2}\left|s_{i j}\right| s_{i j}$

In Eq. (12), $C_{S}$ is the Smagorinsky constant, equal to $0.17, \Delta$ is the filter width (or $I A$ size) and $\left|s_{i j}\right|$ is the characteristic filtered strain rate, defined as $\sqrt{2 s_{i j} s_{i j}}$. Directly substituting the definition of the strain rate tensor and modelled SGS stress tensor into Eq. (10), and expanding into Cartesian coordinates gives the total dissipation rate:

$$
\varepsilon=C_{s}^{2} \Delta^{2}\left\{\begin{array}{l}
2\left(\left(\frac{\partial u}{\partial x}\right)^{2}+\left(\frac{\partial v}{\partial y}\right)^{2}+\left(\frac{\partial w}{\partial z}\right)^{2}+\left(\frac{\partial u}{\partial z}\right)^{2}+\left(\frac{\partial v}{\partial x}\right)^{2}+\left(\frac{\partial v}{\partial z}\right)^{2}+\left(\frac{\partial w}{\partial x}\right)^{2}+\left(\frac{\partial w}{\partial y}\right)^{2}\right\}^{3 / 2} \\
+2\left(\left(\frac{\partial u}{\partial y}\right)\left(\frac{\partial v}{\partial x}\right)+\left(\frac{\partial u}{\partial z}\right)\left(\frac{\partial w}{\partial x}\right)+\left(\frac{\partial v}{\partial z}\right)\left(\frac{\partial w}{\partial y}\right)\right)
\end{array}\right)^{3}
$$

The advantage of stereo-PIV is that it provides the tangential velocity, which cannot be inferred from a standard 2-D PIV setup. However, derivatives of quantities in the out-of-plane $z$-direction are still unknown, yet they are required to form two crossproduct gradients in Eq.(13). Khan (2005) found the contribution of the known crossproduct term $\left(\frac{\partial u}{\partial y}\right)\left(\frac{\partial v}{\partial x}\right)$ to be close to zero for a PBT. In the current study, $\left(\frac{\partial v}{\partial z}\right)\left(\frac{\partial w}{\partial y}\right)$ evaluated from data at Position 3 (see Fig. 2), was also found to be 
negligible. Consequently all three cross-products were neglected from the calculation of $\varepsilon$. A scaling factor of $12 / 10$ was then employed to estimate the remaining squared gradients. The final approximate relationship may then be expressed as:

$$
\varepsilon=C_{s}^{2} \Delta^{2} \overline{\left\{\frac{12}{10}\left(2\left(\frac{\partial u}{\partial x}\right)^{2}+2\left(\frac{\partial v}{\partial y}\right)^{2}+2\left(\frac{\partial w}{\partial z}\right)^{2}+\left(\frac{\partial u}{\partial y}\right)^{2}+\left(\frac{\partial v}{\partial x}\right)^{2}+\left(\frac{\partial w}{\partial x}\right)^{2}+\left(\frac{\partial w}{\partial y}\right)^{2}\right)\right\}^{3 / 2}}
$$

Note that the scaling rule is only appropriate when the known gradient terms are almost equal in magnitude and distribution. In the present case the tangential velocity gradients were considerably greater than the axial and radial counterparts (Unadkat, 2010), nevertheless simple scaling was employed in the absence of any better approximation.

The result is presented in Fig. 12, where $\varepsilon_{\max }$ is $\sim 1.76 N^{3} D^{2}$. Intense dissipation is confined to the blade edge $(r / T=0.156)$, where all velocity gradients, especially the tangential component, had their maximum values. Simple scaling from a 2-D PIV approximation would not have accounted for the unusually high values of the tangential velocity gradients. Similarly, the scaling employed for the out-of-plane gradients could also provide an underestimate.

The maximum dissipation rate obtained from the LES method was found to be an order of magnitude greater than both dimensional analysis methods (not shown here for brevity, but discussed by Unadkat (2010)). Somewhat surprisingly, Sheng et al. (2000) found that the LES analogy corresponded reasonably well with their dimensional analysis case $\left(\varepsilon_{\max } \approx 0.006 N^{3} D^{2}\right.$ for both). It is worth noting that their PIV resolution was $5 \mathrm{~mm}$, resulting in an equally large filter width for the large eddy analogy. Nevertheless, they claimed that the large-eddy PIV approach is inherently more accurate for inhomogeneous and anisotropic flows, such as the present case. The spatial distribution between the dimensional analysis and large eddy analogy methods was also very different. The large eddy approach depends on the large velocity gradients in the vicinity of the impeller, which rapidly decrease in the main flow direction, whereas dimensional analysis produced a more even distribution of $\varepsilon$ along the discharge stream. The comparison between the LES result and dimensional analysis is in agreement with the findings of Kilander and Rasmuson 
(2005), who obtained a five-times greater mean dissipation rate from the LES analogy, and also noted a significant difference in distribution between the two methods.

An interesting result is the "spottiness" of the distribution in Fig. 12, corresponding to regions of intense dissipation. These estimates may have been influenced by a small number of very high velocity gradients in the averaging process. The gradients exist locally, and do not seem to be representative of the surrounding flow. This issue will be considered next, as it is not known if these extreme results are caused from genuinely high velocity gradients arising from turbulence intermittency, or if they are artefact.

\section{Turbulence intermittency and vector filtering}

Spurious velocity vectors may result from lost particle pairs due to in-plane and outof-plane motion, or low local seeding density which in turn leads to poor correlation signal strength. When spurious vectors are produced, the highest correlation peaks are a result of the random pairing of particle images producing the greatest signal.

Fig. 13 shows the time-history of the instantaneous dissipation rate from the LES analogy of Sheng et al. (2000), at a point in the discharge stream over PIV measurements from 500 image pairs. The profile displays some extreme fluctuations with the dissipation rate reaching a maximum of $250 N^{3} D^{2}$. Although these high values are infrequent, they contribute significantly to the mean value of $1.76 N^{3} D^{2}$, which would be significantly lower without their presence. Results like these are responsible for the 'spotty' distribution observed in Fig. 12. Outlying vectors generating high velocity gradients could be eliminated using vector validation filters if assumed to be erroneous, or retained if considered to be genuine fluctuations arising from turbulence intermittency.

In the current study, velocity fields were processed using three variations of the standard deviation filter (considered to be the most comprehensive type) and the dissipation rate was re-calculated. The filters evaluate vectors against the velocity mean of the surrounding points, and eliminate them if they exceed a specified tolerance, i.e. a few standard deviations from the mean. These statistics could be obtained globally (over the entire velocity field), locally (around neighbouring vectors 
of a specific point), or temporally (over sequential PIV measurements at a fixed node). The tolerance was set to three standard deviations in all filters, which is the default level used by TSI Insight vector processing software. All three types altered the dissipation rate in a different way;detailed results are shown in Unadkat (2010). For instance, the global and temporal filters significantly affected the magnitude and distribution, whereas the local filter eliminated a few outlying points only. Consequently, a method of assessment is required for selecting the most appropriate filter, and to check that genuine fluctuations and spottiness inherent to turbulent flows are not removed. The method discussed here is to represent turbulence intermittency via the multifractal model of Meneveau and Sreenivasan (1991); the distribution of filtered dissipation rate profiles will be compared with results from this model, and the most suitable filter will be selected on this basis.

\subsection{Multiplicative process and cascade theory}

It is well known that small scales of turbulence are intermittent. Since the first observations of Batchelor and Townsend (1949) of strongly intermittent bulges in velocity derivative signals, many models have been proposed to account for smallscale intermittency. The dissipation rate has received the most attention over the last 30 years. Most turbulence models are based on the theory of transfer of kinetic energy from large to small scales in a self-similar cascade, with an associated multiplicative process.

In the multiplicative process, large eddies break down into smaller eddies sequentially. Each eddy is given a characteristic linear dimension $r(n)$, where $n$ is the generation step. At each stage, the characteristic scale may be estimated as the product of $n$ numbers called 'length multipliers $l_{j}$ ', which are ratios of consecutive length scales. Hence the characteristic linear dimension is given by:

$$
r(n)=r(0) \prod_{j=i}^{n} r(j) / r(j-1)=r(0) \prod_{j=1}^{n} l_{j}
$$

Secondly, when an eddy decays, the smaller eddies are assumed to receive a fraction of the total dissipation rate $E_{t}$, equal to $E_{r}$. Similar to the characteristic scales, the total dissipation on an eddy of size $r(n)$ may be given in terms of the 
product of $n$ numbers, referred to as the 'measure multipliers $M_{j}$ ', which are ratios of consecutive measures.

$$
E_{r(n)}=E_{r(0)} \prod_{j=i}^{n} E_{r(j)} / E_{r(j-1)}=E_{r(0)} \prod_{j=1}^{n} M_{j}
$$

Thus a multiplicative process may be considered as the disintegration of a large eddy into smaller ones, with each smaller eddy receiving a fraction of the measure of the larger unit, which is the product of the multipliers $l_{j}$ and $M_{j}$ from previous stages. The multipliers are considered to be random variables, with a certain probability distribution. When the distributions are independent of the level or generation step $j$, self-similarity appears, and power-law behaviour occurs in the moments and distribution function of $E_{r}$.

\subsection{Multifractal model of Meneveau and Sreenivasan}

Meneveau and Sreenivasan (1991) provided a detailed account of the development of cascade models for turbulence intermittency and highlighted their shortcomings. Previous models were oversimplified by assuming that the measure multipliers at a given stage were equal, implying non-intermittency (Kolmogorov's 1941 theory) or that they were non-zero and equal for a fraction of the new eddies ( $\beta$ model).

Meneveau and Sreenivasan (1991) proposed a self-similar binomial model, which assumes that the multiplicative process occurs in one dimension. An initial eddy of size $L$ breaks down into two equal segments of size $L / 2$, yielding a length multiplier $l_{j}=1 / 2$. The distribution of energy over eddies is assumed to be unequal, by assigning the measure multipliers a bimodal distribution. The authors found that spatial fluctuations of the dissipation rate could be well quantified by the multipliers $M_{1}=p_{1}=0.7$ and $M_{2}=p_{2}=0.3$. These correspond to the simplest possible fluctuations that reproduce most of the experimental observations. Whether a multiplier takes the value 0.7 or 0.3 is random. However, at each cascade step, a conservation of the measure is imposed such that the sum of $M_{j}$ is always unity. 
The measure of a multiplicative process may be characterised in terms of moments $q$. By introducing an exponent $D_{q}$, the sum of the dissipation rate $E_{r}^{q}$ over disjoint segments of size $r$ may be given as proportional to:

$$
\sum E_{r}^{q} \sim E_{L}^{q}(r / L)^{D_{q}(q-1)}
$$

where $E_{L}$ is the total dissipation contained in an eddy of size $L$. The exponents $D_{q}$ are called 'generalized dimensions'. For the binomial case in particular, the analytical model of $D_{q}$ is given as:

$$
D_{q}=\log _{2}\left\lfloor p_{1}^{q}+p_{2}^{q}\right\rfloor /(1-q)
$$

Anselmet et al. (2001) assessed the fractal nature of the energy distribution at scales $r$, with respect to the entire above mentioned cascade models. They reported that the best predictions were given by the multifractal model at the highest orders, whereas Kolmogorov's 1941 theory and the $\beta$ model departed from the multiplicative process at quite low orders. In light of this, the multifractal model of Meneveau and Sreenivasan (1991) was selected to represent turbulence intermittency in the present case. A full derivation of the model may be found in the paper by Meneveau and Sreenivasan (1991). Only equations necessary for calculating of the generalized dimensions will be repeated in this paper.

\subsection{Measuring $D_{q}$ exponents of dissipation from high-speed PIV data}

It is possible to obtain the multifractal exponents $D_{q}$ at the last cascade step of a multiplicative process, by analysing the dissipation rate at varying degrees of resolution. In the binomial case the process occurs on eddies of size $2^{-n}$ starting at the origin. However in most experiments dissipation is obtained at an unknown level in the multiplicative process, in which case knowledge of the last cascade step, or size and position of eddies at different levels is lacking. Nevertheless, Meneveau and Sreenivasan (1991) showed that it is still possible to use box sizes different from the natural partition $2^{-n}$, and the results remain unaffected.

In practice, the dissipation rate $\varepsilon$ obtained from experimental data at the resolved scale is integrated over disjoint segments of length $r$, yielding dissipation 
$E_{r}$. The segmented dissipation is normalized by the total dissipation in the data set $E_{t}$ :

$E_{r}=\mathrm{\jmath} \varepsilon d r, \quad E_{t}=\mathrm{\jmath} \varepsilon d x$

The scaling range $r$ should be greater than the Kolmogorov length scale $\eta$ and contained within the inertial range where self-similarity appears. Here the distribution functions of the multipliers $l_{j}$ and $M_{j}$ becomes independent of the cascade step (or characteristic size $r$ ). With current PIV limitations, spatial resolutions of the order of the Kolmogorov length scale are not achievable. However, in practice, the data to be analysed is a time series, which using Taylor's frozen turbulence hypothesis may be converted to a length scale, and integrated with respect to $r$. Then the priority is to achieve a temporal resolution of the PIV acquisition rate close to the Kolmogorov time scale. For this analysis, the time series data from high-speed PIV experiments were exploited. Note that in this case, the dissipation rate was calculated using the 2-D LES analogy of Sheng et al. (2000) with a scaling factor of $9 / 5$ to estimate the unknown gradient terms.

To convert the time separation between PIV image pairs $(\Delta t)$ into longitudinal separation $(\Delta r)$, Taylor's hypothesis was applied such that $\Delta r=\bar{u} \Delta t$, where $\bar{u}$ is the time-averaged mean radial velocity at that point in the flow field. The power spectrum of the radial velocity time-series obtained at the point $r / T=0.167, z / T=0.333$ in the discharge stream is presented in Fig. 14. A periodogram estimate of the power spectral density (PSD) was obtained. Note that the wave number is $\kappa=f / \bar{u}$ where $t$ is the sampling frequency of $5 \mathrm{kHz}$. It may be observed that the temporal separation between the double-image frames $\left(2 \times 10^{-4} \mathrm{~s}\right)$ was sufficient to generate a power spectrum with an identifiable inertial sub-range: the solid line in Fig. 14 indicates a $-5 / 3$ slope. The dashed vertical lines enclose the scaling range $-2.25<\log _{10}[\kappa \eta]<-1.5$, corresponding to $32<r / \eta<178$.

The dissipation rate profiles from the filtered and unfiltered velocity fields were integrated over arbitrary disjoint segments of length $r$ within the scaling range. The sums $\sum\left(E_{r} / E_{t}\right)^{q}$ for $q$ values between 0 and +5 were computed. Relevant 
quantities in determining the $D_{q}$ exponents are the logarithm of the moments divided by $(q-1)$. Then, log-log plots of $\left[\sum\left(E_{r} / E_{t}\right)^{q}\right]^{1 /(q-1)}$ as a function of $r / \eta$ were constructed for various $q$ values, where the slope of the linear regions provided the $D_{q}$ exponents.

The log-log plot of $\left[\sum\left(E_{r} / E_{t}\right)^{q}\right]^{1 /(q-1)}$ versus $r / \eta$ of the globally-filtered profile for $-5<q<+5$ is shown in Fig. 15. Power-law behaviour cannot be identified unambiguously in the plots for $q<0$. A similar result was observed for the other filtered and unfiltered cases. Consequently these moments were excluded from further analysis. Meneveau and Sreenivasan (1991) suggested that using data sets of length of the order $\sim 100 \Lambda_{u}$ (where $\Lambda_{u}$ is the integral length scale) improves the scaling. Secondly, averaging results over tens of such samples improves the statistical convergence of $D_{q}$ considerably. These features enable computation of $D_{q}$ for $q<0$. The data length in the current study was found to be only $\sim 130 \Lambda_{u}$, hence averaging over multiple samples was not possible. This explains the poor power-law behaviour for the negative $q$ range. The authors stated that high positive values of $q$ emphasize regions of intense dissipation, whereas negative $q$ values accentuate low dissipation regions. In this analysis, the aim is to quantify the nature of the intense dissipation, so the negative $q$ range is unimportant.

The moment exponents $D_{q}$ obtained from the slopes of the lines between $0<q<+5$ for all four profiles are plotted in Fig. 16, against the analytical model of Meneveau and Sreenivasan (1991). It may be observed that the generalized dimensions from the unfiltered profile depart significantly from the multifractal model, indicating that the intense fluctuations observed in Fig. 13 were not genuine intermittency. Instead they arise from velocity gradients of spurious vectors. The removal of this noisy data necessitates the application of a type of standard deviation filter. The generalized dimensions of the filtered profiles are much closer to the analytical model. The result is surprising since the $D_{q}$ exponents were obtained from a single sample and no averaging was performed to improve the statistics. The result suggests that all three filters appear adequate in removing the spurious vectors, and 
produce a more plausible illustration of intermittency. The $D_{q}$ exponents from the globally filtered profile agree most with the multifractal model, indicating that this is the best filter for the present case. The result may be surprising since the global field contains a wide range of velocities, yielding a large standard deviation.

The dissipation rate in the entire field of view, after applying the global standard deviation filter, is portrayed in Fig. 17. To further validate the efficiency of the global filter, the instantaneous dissipation rate profile obtained at the point of maxima ( $r / T=0.158, z / T=0.331)$ was also validated against the multifractal model. The generalised dimensions arising from this analysis are shown in Fig 18. The $D_{q}$ exponents from this analysis coincide exactly with the model of Meneveau and Sreenivasan (1991), providing confidence in the filter choice as well as the maximum dissipation rate estimate. In light of this finding, the stereo-PIV results discussed in the proceeding section have been processed using the global standard deviation filter.

\section{Ratios of maximum and mean energy dissipation}

The mean dissipation rate is often used in the scale up of stirred vessels; however it provides no information about the distribution or maximum value throughout the tank. The maximum dissipation rate may deviate significantly from the mean, which may determine, for instance, the minimum drop size in dispersed gas or liquid flows (Zhou and Kresta, 1996), and the rate of fines generation in nano-particle dispersions (Xie et al., 2008). After processing the stereo-PIV velocity fields using a global standard deviation filter, and applying the LES analogy of Sheng et al. (2000) to the 3-D data, $\varepsilon_{\max }$ was found to be $\sim 0.17 N^{3} D^{2}$ at $1500 \mathrm{rpm}$. The mean dissipation rate was $0.004 N^{3} D^{2}$, giving $\varepsilon_{\max } / \bar{\varepsilon} \approx 40$. The sawtooth impeller is a high shear mixing device, so a large maximum-to-mean ratio was to be expected. It is of interest to compare this ratio with other impeller types, to evaluate their relative order of magnitude. The mean and maximum dissipation rates cited in literature are summarised in Table 1, and $\varepsilon_{\max } / \bar{\varepsilon}$ calculated from this data. There is a significant variation in the reported values of $\varepsilon_{\max } / \bar{\varepsilon}$ even between common impeller types. Three main factors contribute to these variations: differences in tank geometry, flow measurement technique and dissipation rate calculation method. 
Zhou and Kresta (1996) employed 1-D LDA to measure flow velocities generated by a PBT with $D / T=0.333$. They used dimensional analysis to obtain the dissipation rate and found $\varepsilon_{\text {max }} / \bar{\varepsilon} \approx 38$. Khan (2005) used PIV and employed the 2-D LES analogy resulting in $\varepsilon_{\max } / \bar{\varepsilon} \approx 118$. Since the impeller geometry was identical in both studies, the difference must be due to the calculation method. Note in the present case, $\varepsilon_{\max }$ from dimensional analysis was an order of magnitude smaller than the LES method. However Sheng et al. (2000) found $\varepsilon_{\text {max }} / \bar{\varepsilon} \approx 8$ from their LES analogy for a PBT, which is surprisingly smaller than the dimensional result of Zhou and Kresta (1996). In fact Sheng et al. (2000) found that both LES and dimensional analysis yielded similar results. Their PIV resolution was $5 \mathrm{~mm}$, compared to only $1.08 \mathrm{~mm}$ in Khan's (2005) study. In the LES method, turbulence statistics at scales smaller than the PIV resolution are filtered and modelled, so the low result is not unexpected when taking this into account.

All RDT studies listed in Table 1 were carried out using $D / T=0.333$. The lowest result was obtained from the direct evaluation of Sharp and Adrian (2001) to be $\varepsilon_{\text {max }} / \bar{\varepsilon} \approx 16$, at a PIV resolution of $0.5 \mathrm{~mm}$. Baldi and Yianneskis (2004) also employed direct calculation of $\varepsilon$ for various $R e$ flows using a PIV resolution of $0.1 \mathrm{~mm}$. The authors noted that the Kolmogorov length scale decreases in the most turbulent regions as $R e$ increases; hence the accuracy of $\varepsilon$ decreases in the vicinity of the impeller, at a fixed PIV resolution for direct evaluation methods. The most reliable measure was assumed to be at $\operatorname{Re}=2 \times 10^{4}$, where $\varepsilon_{\max } / \bar{\varepsilon} \approx 57$. As a final consideration, note the various power numbers $P_{o}$ used to estimate the mean dissipation rate. The power number (hence $\bar{\varepsilon}$ ) of RDTs is dependent on Reynolds number up to $R e \sim 7 \times 10^{4}$ (Chapple et al., 2002) or up to $R e \sim 6 \times 10^{4}$ as found by Khan (2005). Since the listed experiments were carried out in varying $R e$ flows below $R e \sim 6 \times 10^{4}, \varepsilon_{\max } / \bar{\varepsilon}$ is expected to deviate to some extent, even for identical $\varepsilon_{\max }$ estimates, due to the differences in $\bar{\varepsilon}$. For instance, the $\varepsilon_{\max }$ estimates from dimensional analysis of Zhou and Kresta (1996) and TKE balance of Escudie and Line (2003) are greater than the direct calculations. However since both employ 
higher $P_{o}$ values for the same impeller, the final $\varepsilon_{\max } / \bar{\varepsilon}$ ratios are smaller: 48 and 45 respectively.

It has been found that $\varepsilon_{\max } / \bar{\varepsilon} \approx 40$ for the EkatoMizer discharge stream is within the range of ratios reported for PBT and RDT impellers. Therefore it is debatable whether the EkatoMizer really sustains a high shear advantage, because of its geometric design. Note that before calculating the dissipation rate, a global standard deviation filter was applied to the velocity field for validation, which eliminated some large magnitude vectors. This reduced the maximum dissipation rate significantly, from $\varepsilon_{\max } \approx 1.76 N^{3} D^{2}$ to $\varepsilon_{\max } \approx 0.17 N^{3} D^{2}$ at $1500 \mathrm{rpm}$. It is believed that the global standard deviation filter provides a more realistic representation of turbulence intermittency, in line with the multifractal model of Meneveau and Sreenivasan (1991). Therefore the ratio obtained after filtering is accepted as the best estimate in the present case. It follows that PIV experiments reported previously in the literature may be subjected to the same argument, in that their data could possibly benefit from filtering. Since vector validation techniques have such a profound impact on the dissipation rate results, particularly using gradient-based calculation methods, a comprehensive comparison of $\varepsilon_{\max } / \bar{\varepsilon}$ is difficult to achieve.

A hydrofoil impeller was studied by Baldi and Yianneskis (2003), with PIV resolution between 1.25 and $0.111 \mathrm{~mm}$. Direct estimates of the dissipation rate gave $0.02<\varepsilon_{\max } / N^{3} D^{2}<0.8$ with corresponding $2<\varepsilon_{\max } / \bar{\varepsilon}<80$, emphasizing the importance of resolution. Kilander and Rasmuson (2005) also investigated a hydrofoil using PIV and a modified large eddy method to obtain the dissipation rate. Their results indicated $\varepsilon_{\max } / \bar{\varepsilon} \approx 921$, which is the greatest value reported yet for any mixer. This is especially surprising for a hydrofoil which is thought of as a low-shear device. Besides the issue of vector validation, the large magnitude of $\varepsilon_{\max } / \bar{\varepsilon}$ may be attributed to the fact that the authors incorrectly took the space between adjacent vectors (there was $75 \%$ overlap) to be the filter width; it should be equal to the $I A$ length, regardless of the overlap between interrogation areas.

On a final note it should be emphasised that the ratio $\varepsilon_{\max } / \bar{\varepsilon}$ has only been evaluated from PIV results obtained within the EkatoMizer impeller stream. In 
separate works (Unadkat, 2010), a KE balance around the impeller region suggested that the maximum dissipation rate actually occurs inside the impeller swept volume, i.e. in a region which cannot be accessed by PIV, since the EkatoMizer blades completely obstruct the field of view. The total KE balance revealed that $\varepsilon_{\max } / \bar{\varepsilon}$ could be as high as 273 in the swept volume. If this is true then the EkatoMizer could be considered as a high shear impeller. However dissipation rate estimates would also be required in the swept volume of the other impellers to enable an equivalent assessment.

\section{Conclusions}

The flow field generated by an EkatoMizer in the vicinity of the sawtooth blades and in the discharge stream has been studied using stereoscopic and high-speed PIV techniques. An analysis of the mean flow field elucidated the general structure of fluid drawn into the impeller region axially and discharged radially; the latter characterised the impeller stream. Flow anisotropy was determined via the anisotropy tensor which is characterized using the turbulent Reynolds stresses, and showed that the flow was anisotropic close to the blade, and returned to isotropy further away from the impeller. Instantaneous vector plots revealed vortices in the discharge stream, which were interpreted as low-frequency phenomena (relative to the blade passage) with frequencies in the range $0-200 \mathrm{~Hz}$. Following the methodology of Fan et al. (2004), and performing a spatio-temporal analysis of the vorticity, macro-instabilities were found to have a high probability of occurrence in the discharge stream, which coincided with the location of observed vortex structures in the instantaneous vector plots. The macro-instabilities in the current study are very different from the traditionally described structures reported in literature for RDT and PBT impellers, which usually form behind baffles, circulate around the impeller shaft, and persist near the liquid surface. Additionally, the Ml frequencies reported for the EkatoMizer discharge stream are approximately two orders of magnitude greater than for RDT and PBTs. The results indicate the possibility of characterising another form of low-frequency phenomena

After considering various other methods (Unadkat, 2010), the LES analogy of Sheng et al. (2000) was employed to estimate the dissipation rate, as it accounts for three-dimensionality of the flow by incorporating all three velocity components (all 
available using stereo PIV), and represents the turbulence at the smaller unresolved scales using a SGS model. The resulting 'spotty' distribution of $\varepsilon$ was thought to arise from extremely large velocity gradients resulting from erroneous vectors or from turbulence intermittency. The multifractal model of Meneveau and Sreenivasan (1991) was used as a basis of comparison to successfully distinguish between spurious PIV results and genuine turbulence intermittency. By extending this method to analyse filtered velocity fields, the global standard deviation filter was found to be the most suitable type in its class for vector validation. The recommendation is that the best filter should be selected on a case-by-case basis, since this is largely dependent on the degree of turbulence and inhomogeneity of the flow field, as well as experimental variables such as the seed tracer number density and time-delay between image frames, which in turn influence the cross-correlation procedures. After selecting the best filter, the multifractal model may also be used to optimise filter parameters, such as tolerance level of the global filter or neighbourhood size of the local filter. In all cases, the generalised dimensions calculated from the filtered data were in much better agreement with the multifractal model than the $D_{q}$ values obtained from the unfiltered data. Careful filtering of the raw velocity data is therefore essential in using gradient-based methods to determine dissipation rates.

Finally, a comparison of the $\varepsilon_{\max } / \bar{\varepsilon}$ ratio of the EkatoMizer with other common impellers such as PBTs and RDTs suggest that its does not produce particularly high turbulent shear rates in the discharge stream. It was noted, however, that PIV experiments were only conducted outside the sawtooth blades and it is possible that the maximum dissipation rate occurs within the impeller swept volume, making $\varepsilon_{\max } / \bar{\varepsilon}$ significantly higher. 


\section{Nomenclature}

\section{Roman letters}

$a_{i j} \quad$ Anisotropy tensor

$b_{i j} \quad$ Normalized anisotropy tensor

B Blade thickness

C Impeller clearance from vessel base

$C_{s} \quad$ Smagorinsky constant

D Impeller diameter

$D_{q} \quad$ Generalized dimension in multifractal model

$E_{r} \quad$ Fraction of dissipation on secondary eddy of length $r(n)$

$E_{t} \quad$ Total dissipation in a primary eddy $\left[\mathrm{m}^{2} \mathrm{~s}^{-3}\right]$

$t \quad$ Frequency

$F(f) \quad$ Normalized PSD

$h(f) \quad$ None-normalized PSD from a periodogram estimate

$H \quad$ Water fill height

$k \quad$ Turbulent kinetic energy

$l \quad$ Characteristic eddy size

$l_{j} \quad$ Length multiplier

$M_{j} \quad$ Measure multiplier

$N \quad$ Impeller speed

$P_{o} \quad$ Power number of impeller 
$P(f) \quad$ Spectral distribution function

$q \quad$ Moments of generalized dimension $D_{q}$

$r(n) \quad$ Characteristic linear dimension of eddy from generation step $n$

Re Reynolds number

$s_{i j} \quad$ Resolved scale strain rate tensor

$\left|s_{i j}\right| \quad$ Characteristic filtered strain rate

$T \quad$ Vessel diameter

$u_{i} \quad$ Instantaneous fluid velocity in Cartesian direction $i$

$\overline{u_{i}} \quad$ Mean fluid velocity in Cartesian direction $i$

$u_{i}^{\prime} \quad$ Fluctuating fluid velocity in Cartesian direction $i$

$x_{i} \quad$ Cartesian coordinate in direction $i$

$X_{i} \quad$ Position in the image plane in direction $i$

\section{Greek symbols}

$\delta_{i j} \quad$ Kronecker delta

$\Delta \quad$ Filter width in large eddy simulation analogy

$\Delta r \quad$ Longitudinal separation between data pints

$\Delta t \quad$ Time between two PIV double-image frame exposures

$\varepsilon \quad$ Turbulent kinetic energy dissipation rate

$\bar{\varepsilon} \quad$ Average turbulent kinetic energy dissipation rate

$\left[\mathrm{m}^{2} \mathrm{~s}^{-3}\right]$

$\varepsilon_{\max } \quad$ Maximum dissipation rate 
$\overline{\varepsilon_{S G S}} \quad$ Reynolds averaged sub-grid scale dissipation rate

$\left[\mathrm{m}^{2} \mathrm{~s}^{-3}\right]$

$\eta \quad$ Kolmogorov length scale

$\kappa \quad$ Wave number

$\Lambda_{u_{i}} \quad 2-\mathrm{D}$ integral length scale from autocorrelation of the velocity $u_{i}$

$\tau_{i j} \quad$ Sub-grid scale stress tensor

$\left[\mathrm{N} \mathrm{m}^{-2}\right]$

\section{Abbreviations}

CCD Charge coupled device

IA Interrogation area

LDA/V Laser Doppler anemometry/velocimetry

LES Large eddy simulation

Nd:YAG Neodym-Yttrium-Aluminium Garnet

PBT Pitched blade turbine

PIV Particle image velocimetry

PSD Power spectral density

RDT Rushton disc turbine

SGS Sub-grid scale

TKE Turbulent kinetic energy

$\left[\mathrm{m}^{2} \mathrm{~s}^{-2}\right]$ 


\section{References}

Anselmet, F., Antonia, R. A. and Danaila, L., (2001), Turbulent flows and intermittency in laboratory experiments, Planetary and Space Science, 49, 1177-1191.

Baldi, S. and Yianneskis, M., (2003), On the direct measurement of turbulence energy dissipation in stirred vessels with PIV, Ind. Eng. Chem. Res., 42, 70067016.

Baldi, S. and Yianneskis, M., (2004), On the quantification of energy dissipation in the impeller stream of a stirred vessel from fluctuating velocity gradient measurements, Chem. Eng. Sci., 49, 2659-2671.

Batchelor, G. K. and Townsend, A. A., (1949), The nature of turbulent motion at large wave-numbers, Proc. R. Soc. London Ser. A-Mathematical and Physical Sciences, 199(1057), 238-255.

Bittorf, K. J. and Kresta, S. M., (1999), Active volume of mean circulation for stirred tanks agitated with axial impellers, Chem. Eng. Sci., 55, 1325-1335.

Chapple, D., Kresta, S. M., Wall, A. and Afacan, A., (2002), The effect of impeller and tank geometry on power number for a pitched blade turbine, Chem. Eng. Res. Des., 80(A4), 364-372.

Clark, R. A., Ferziger, J. H. and Reynolds, W. C., (1979), Evaluation of subgrid-scale models using an accurately simulated turbulent flow, J. Fluid Mech., 91, 1-16.

Ducci, A. and Yianneskis, M., (2005), Direct determination of energy dissipation in stirred vessels with two-point LDA, AIChE J., 51(8), 2133-2148.

Derksen, J. J., Doelman, M. S. and Van den Akker, H. E. A., (1999), Threedimensional LDA measurements in the impeller region of a turbulently stirred tank, Exp. Fluids, 27(6), 522-532.

Escudie, R. and Line, A., (2003), Experimental analysis of hydrodynamic in a radially agitated tank, AlChE J., 49(3), 585-603. 
Fan, J., Rao, Q., Wang, Y. and Fei, W., (2004), Spatio-temporal analysis of macroinstability in a stirred vessel via digital particle image velocimetry (DPIV), Chem. Eng. Sci., 59, 1863-1873.

Gabriele, A., Nienow, A. W. and Simmons, M. J. H., (2009), Use of angle resolved PIV to estimate local specific energy dissipation rates for up- and downpumping pitched blade agitators in a stirred tank, Chem. Eng. Sci., 64(1), 126143.

Guillard, F., Tragardh, C. and Fuchs, L., (2000), A study of turbulent mixing in a turbine-agitated tank using a fluorescence technique, Exp. Fluids, 28, 225-235.

Haam, S., Brodky, R. S. and Fasano, B., (1992), Local heat transfer in a mixing reactor using the heat flux sensors, Ind. Eng. Chem. Res., 31, 1384-1391.

Hasal, P., Montes, J. L., Boisson, H. C. and Fort, I., (2000), Macro-instabilities of velocity field in stirred vessel: detection and analysis, Chem. Eng. Sci., 55(2), 391-401.

Khan, F. R., (2005), Investigation of turbulent flows and instabilities in a stirred vessel using Particle Image Velocimetry, PhD thesis, Loughborough University, Loughborough, UK.

Khan, F. R., Rielly, C. D. and Hargrave, G. K., (2004), A multi-block approach to obtain angle-resolved PIV measurements of the mean flow and turbulence fields in a stirred vessel, Chem. Eng. Technol., 27(3), 264-269.

Kilander, J. and Rasmuson, A. C., (2005), Energy dissipation and macro instabilities in a stirred square tank investigated using an LE PIV approach and LDA measurements, Chem. Eng. Sci., 60, 6844-6856.

Kolmogorov, A. N., (1941), The local structure of turbulence in incompressible viscous fluid for very large Reynolds number, Dokl. Akad. Nauk SSSR, 30, 299303. 
Kresta, S. M. and Wood, P. E., (1993), The flow field produced by a pitched blade turbine: characterization of the turbulence and estimation of the dissipation rate, Chem. Eng. Sci., 48, 1761-1774.

Lee, K. C. and Yianneskis, M., (1998), Turbulence properties of the impeller stream of a Rushton turbine, AIChE J., 44(1), 13-24.

Lui, S., Meneveau, C. and Katz, J., (1994), On the properties of similarity subgridscale models as deduced from measurements in a turbulence jet, J. Fluid Mech., 275, 83-119.

Lumley, J., (1978), Computational modelling of turbulent flows, Adv. Appl. Mech., 24, 123-176.

Meneveau, C. and Sreenivasan, K. R., (1991), The multifractal nature of turbulent energy dissipation, J. Fluid Mech., 224, 429-484.

Montes, J. L., Boisson, H. C., Fort, I. and Jahoda, M., (1997), Velocity field macroinstabilities in an axially agitated mixing reactor, Chem. Eng. J., 67, 139-145.

Nikiforaki, L., Montante, G., Lee, K. C. and Yianneskis, M., (2003), On the origin, frequency and magnitude of macro-instabilities of the flows in stirred vessels, Chem. Eng. Sci., 58, 2937-2949.

Prasad, A. K., (2000), Stereoscopic Particle Image Velocimetry, Exp. Fluids, 29(2), 103-116.

Pope, S. B., (2000), Turbulent flows, Cambridge University Press, Cambridge.

Saarenrinne, P., Piirto, M. and Eloranta, H., (2001), Experiences of turbulence measurement with PIV, Meas. Sci. Technol., 12, 1904-1910.

Sharp, K. V. and Adrian, R. J., (2001), PIV study of small-scale flow structure around a Rushton turbine, AIChE J., 47(4), 766-778.

Sheng, J., Meng, H. and Fox, R. O., (2000), A large eddy PIV method for turbulence dissipation rate estimation, Chem. Eng. Sci., 55(20), 4423-4434. 
Smagorinsky, J., (1963), General circulation experiments with the primitive equation I the basic experiment, Monthly Weather Review, 91, 99-164.

Unadkat, H., (2010), Investigation of turbulence modulation in solid-liquid suspensions using FPIV and micromixing experiments, $P h D$ Thesis, Loughborough University, Loughborough, UK.

Van't Riet, K. and Smith, J. M., (1975), Trailing vortex system produced by rushton turbine agitators, Chem. Eng. Sci., 30(9), 1093-1105.

Wu, H. and Patterson, G. K., (1989), Laser-Doppler measurements of turbulent-flow parameters in a stirred mixer, Chem. Eng. Sci., 44(10), 2207-2221.

Xie, L., Rielly, C. D., Eagles, W., Özcan-Taskin, G., (2007), Dispersion of nanoparticle clusters using mixed flow and high shear impellers in stirred tanks, Chem. Eng. Res. Des., 85(A5), 676-684.

Xie, L., Rielly, C. D., Özcan-Taskin, G., (2008), Break-Up of Nanoparticle Agglomerates by Hydrodynamically Limited Processes, J. Disper. Sci. Technol., 29(4), 573-589.

Yianneskis, M., Popiolek, Z. and Whitelaw, J. H., (1987), An experimental study of the steady and unsteady flow characteristics of stirred reactors, J. Fluid Mech., $175,537-555$.

Yianneskis, M. and Whitelaw, J. H., (1993), On the structure of the trailing vortices around Rushton turbine blades, Chem. Eng. Res. Des., 71, 534-550.

Zhou, G. and Kresta, S. M., (1996), Impact of tank geometry on the maximum turbulence energy dissipation rate for impellers, AIChE J., 42(9), 2476-2490. 


\section{Captions}

Figure 1: Stereo-PIV setup

Figure 2: Measurement positions

Figure 3: Details of impeller teeth dimensions

Figure 4: Vector plot of mean axial and radial velocity at Position 1, $1500 \mathrm{rpm}$

Figure 5: Vector plot of instantaneous axial and radial velocity at Position 1, 1500 rpm

Figure 6: Mean tangential velocity field obtained at (a) Position 1 and (b) Position 2, $1500 \mathrm{rpm}$

Figure 7: (a) axial (b) radial and (c) tangential rms velocities at Position 1, $1500 \mathrm{rpm}$

Figure 8: TKE from the full definition at Position 1, $1500 \mathrm{rpm}$

Figure 9: Invariant parameters $\xi$ and $\eta$ from 3-D PIV time averaged measurements, Position 1, $1500 \mathrm{rpm}$. Solid lines indicate the Lumley triangle.

Figure 10: PSD of the radial velocity time series at $r / T=0.18, z / T=0.34,1500$ rpm, $5 \mathrm{kHz}$ PIV measurements

Figure 11: Two-dimensional distribution of probability of $\mathrm{Ml}$ occurrence between 0 and $200 \mathrm{~Hz}$, from high-speed PIV measurements at $1500 \mathrm{rpm}, 5 \mathrm{kHz}$

Figure 12: Dissipation rate from the LES analogy of Sheng et al. (2000) using stereoPIV ensemble averaged measurements, Position 1, 1500 rpm

Figure 13: Time-history of instantaneous normalized dissipation rate at a point in the discharge stream, over 500 PIV measurements, 1500 rpm

Figure 14: Power spectrum of the radial velocity at point $r / T=0.167, z / T=0.333$ in the discharge stream, from high-PIV measurements at $5 \mathrm{kHz}$ and $1500 \mathrm{rpm}$

Figure 15: Log-log plot of $\left[\sum\left(E_{r} / E_{t}\right)^{q}\right]^{1 /(q-1)}$ versus $r / \eta$ for several $q$ values, from the dissipation rate profiles obtained after applying the global standard deviation filter 
Figure 16: Moment exponents $D_{q}$ from the analysis of dissipation rate profiles before and after the application of various standard deviation filters. The solid line represents the multifractal model of Meneveau and Sreenivasan (1991).

Figure 17: Dissipation rate from the 2-D LES analogy of Sheng et al. (2000), using high-speed PIV ensemble averaged measurements, after applying a global standard deviation filter to the velocity field

Figure 18: Moment exponents $D_{q}$ from the analysis of the dissipation rate profile at the point of maxima $(r / T=0.158, z / T=0.331)$ in Fig. 15 . The solid line represents the multifractal model of Meneveau and Sreenivasan (1991).

Table 1: Maximum and mean dissipation rates of various impellers reported in literature 


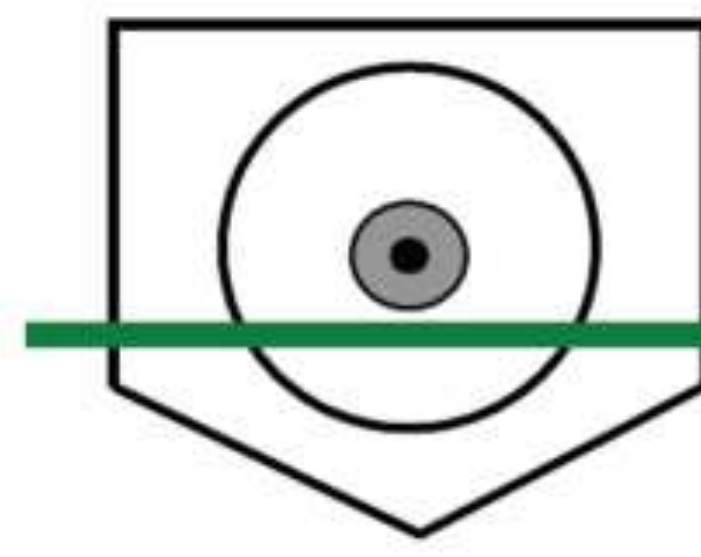

Cylindrical lens

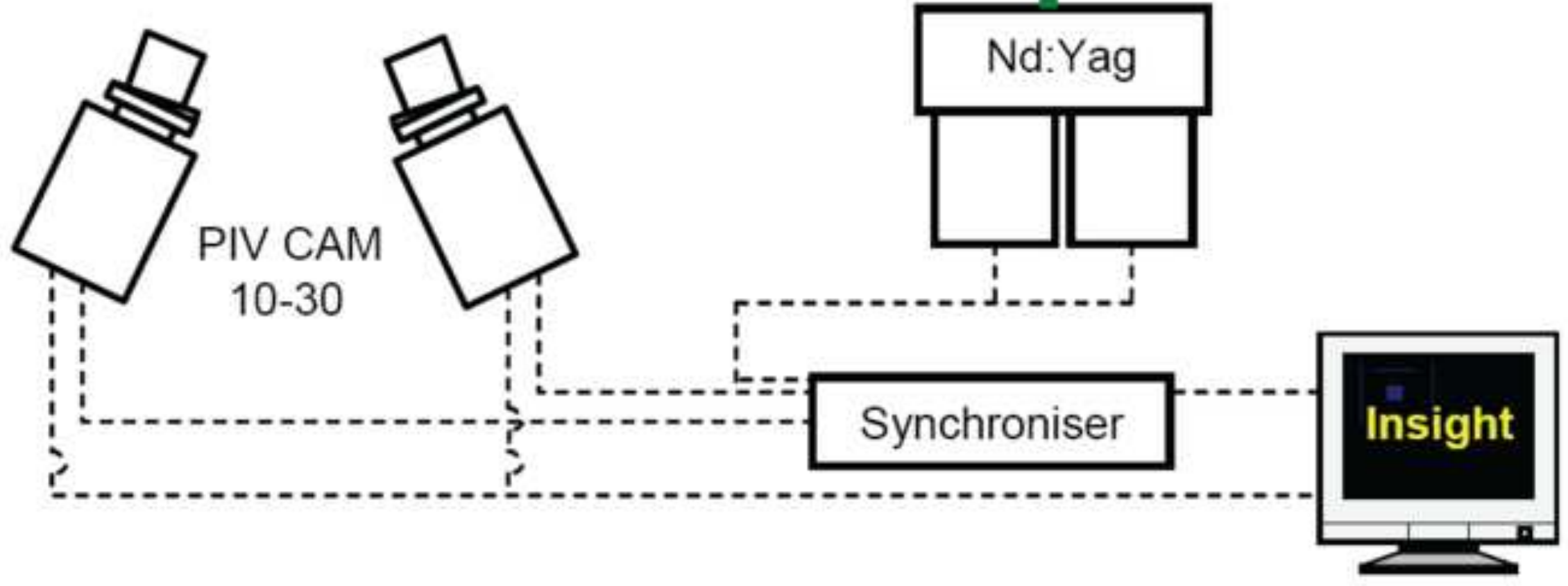



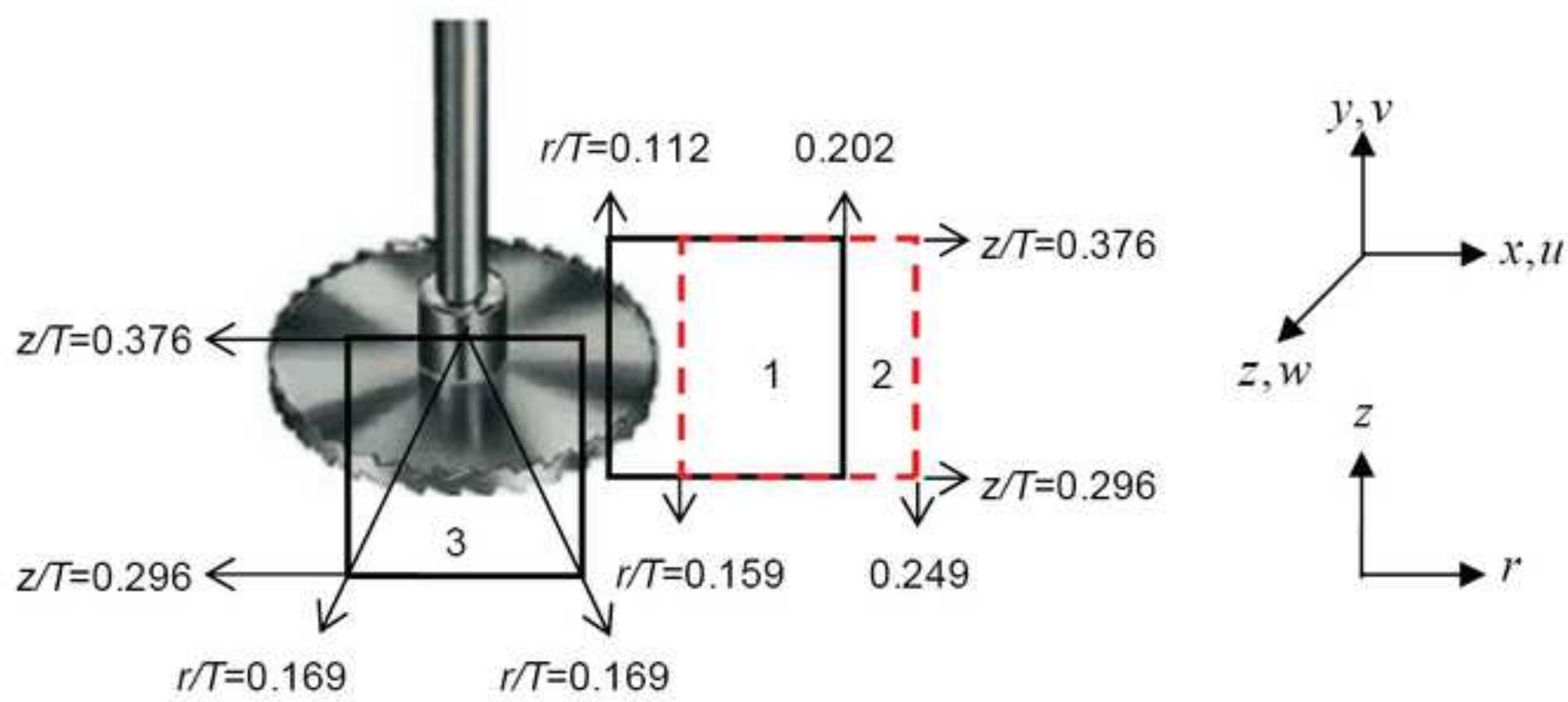

$$
r / T=0.169 \quad r / T=0.169
$$




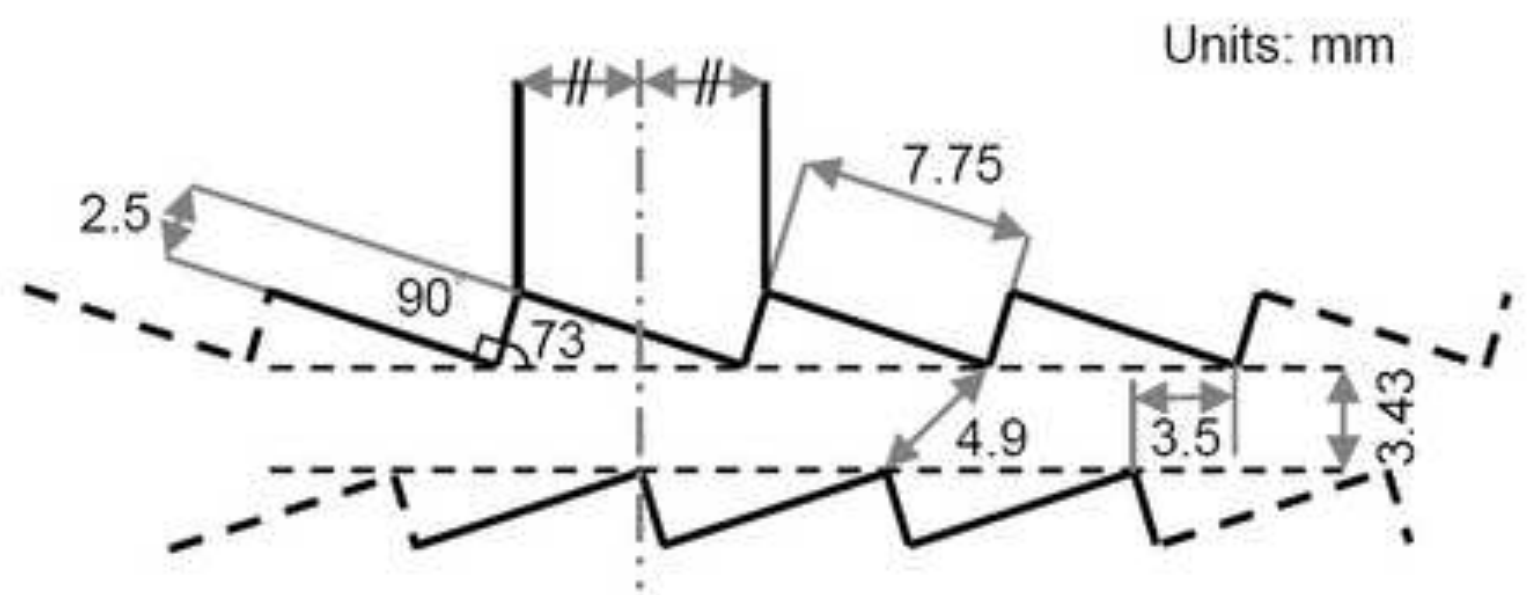

\section{EkatoMizer®}

72 teeth in total: 36 upper + lower Impeller diameter: $95 \mathrm{~mm}$

Thickness of upper teeth: $1.62 \mathrm{~mm}$ Thickness of lower teeth: $1.75 \mathrm{~mm}$ 


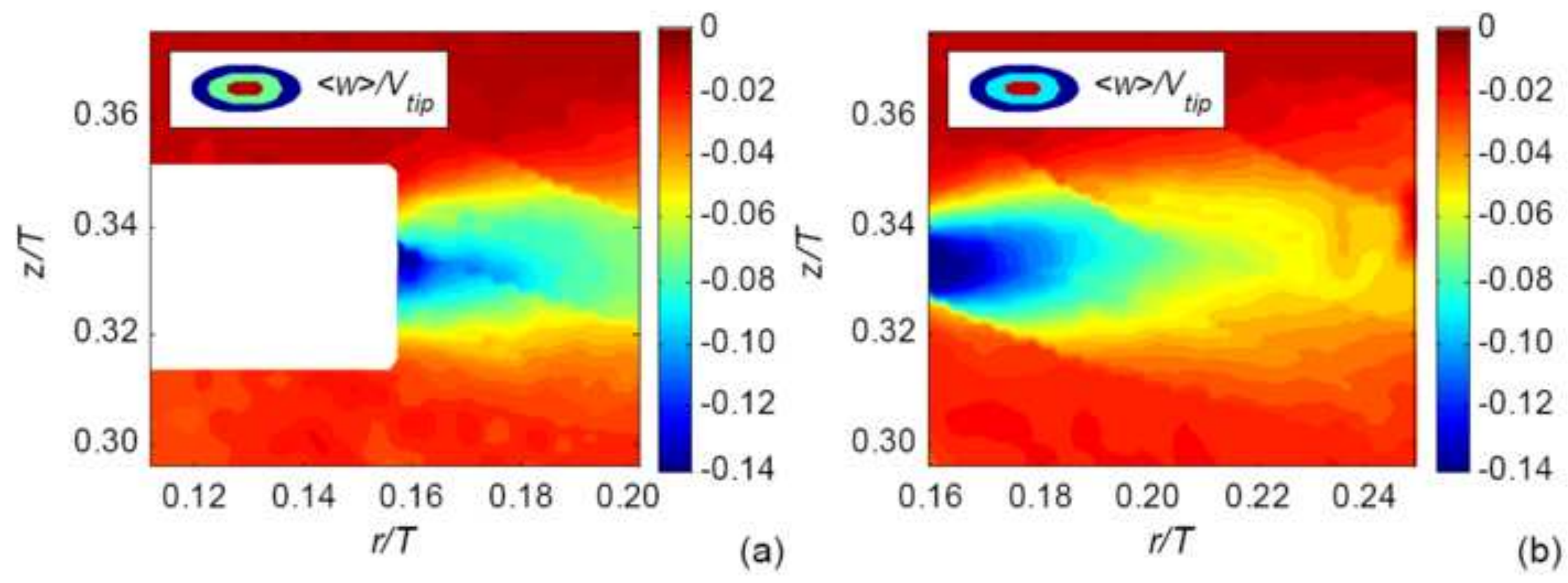



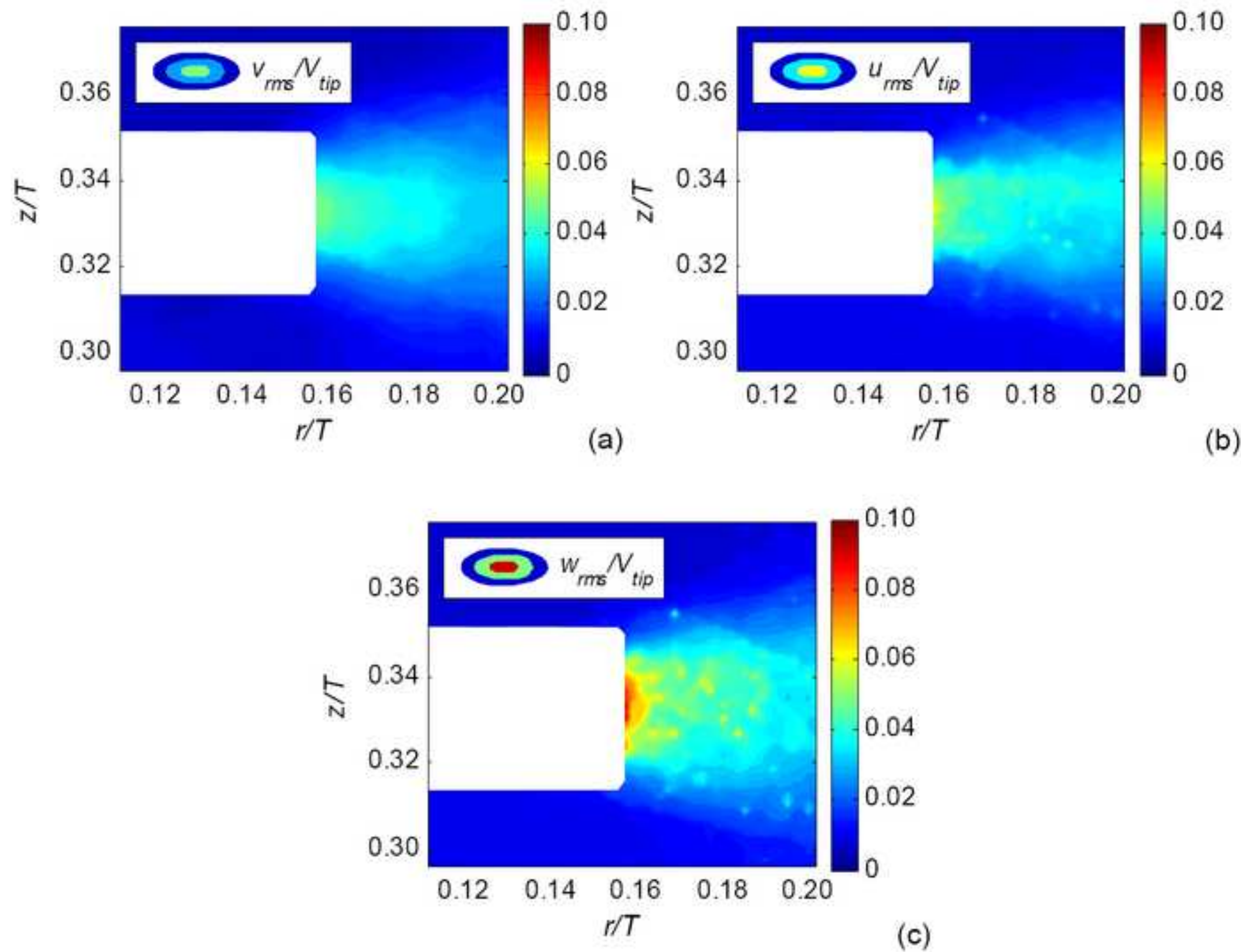

(c) 


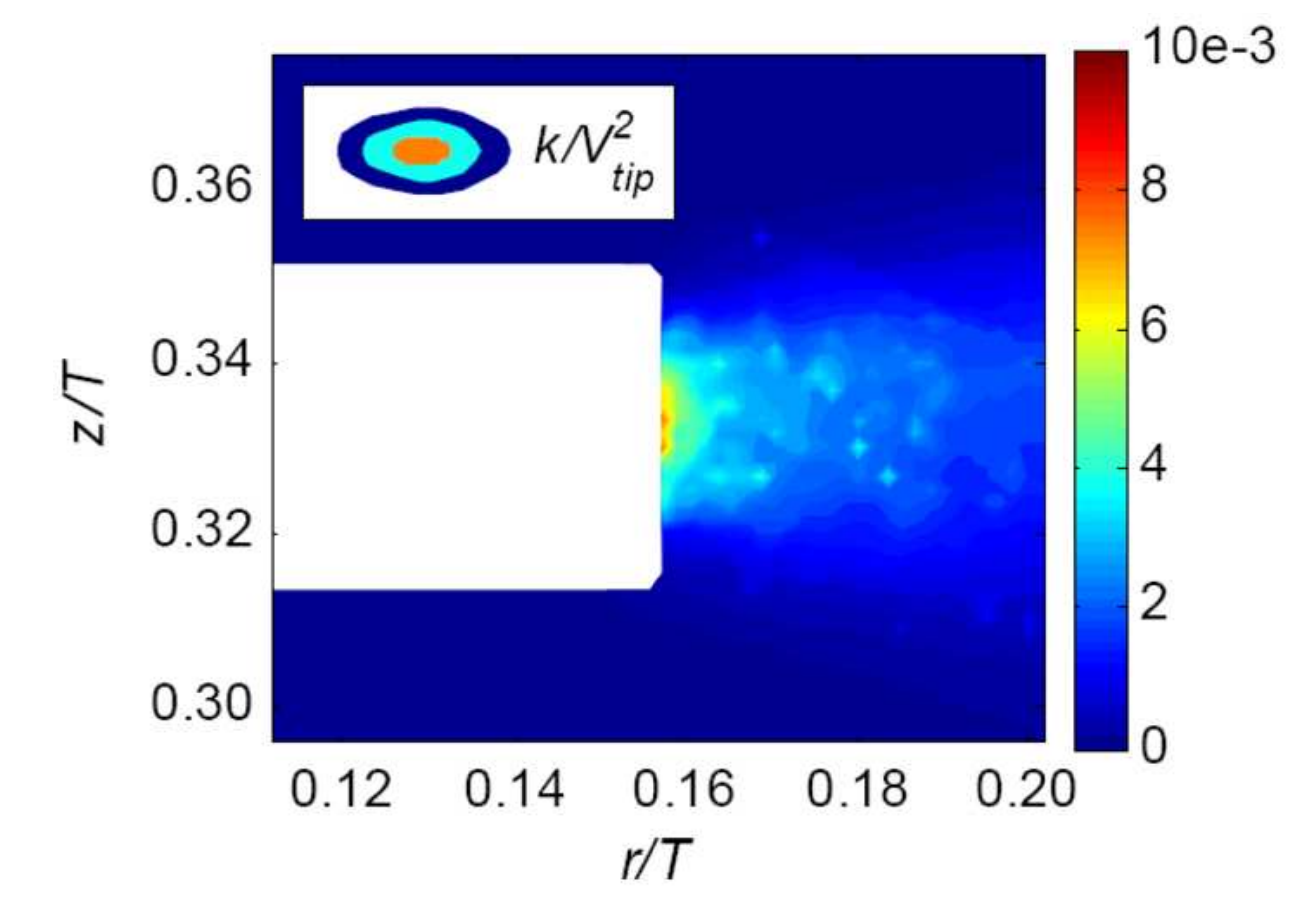
. 

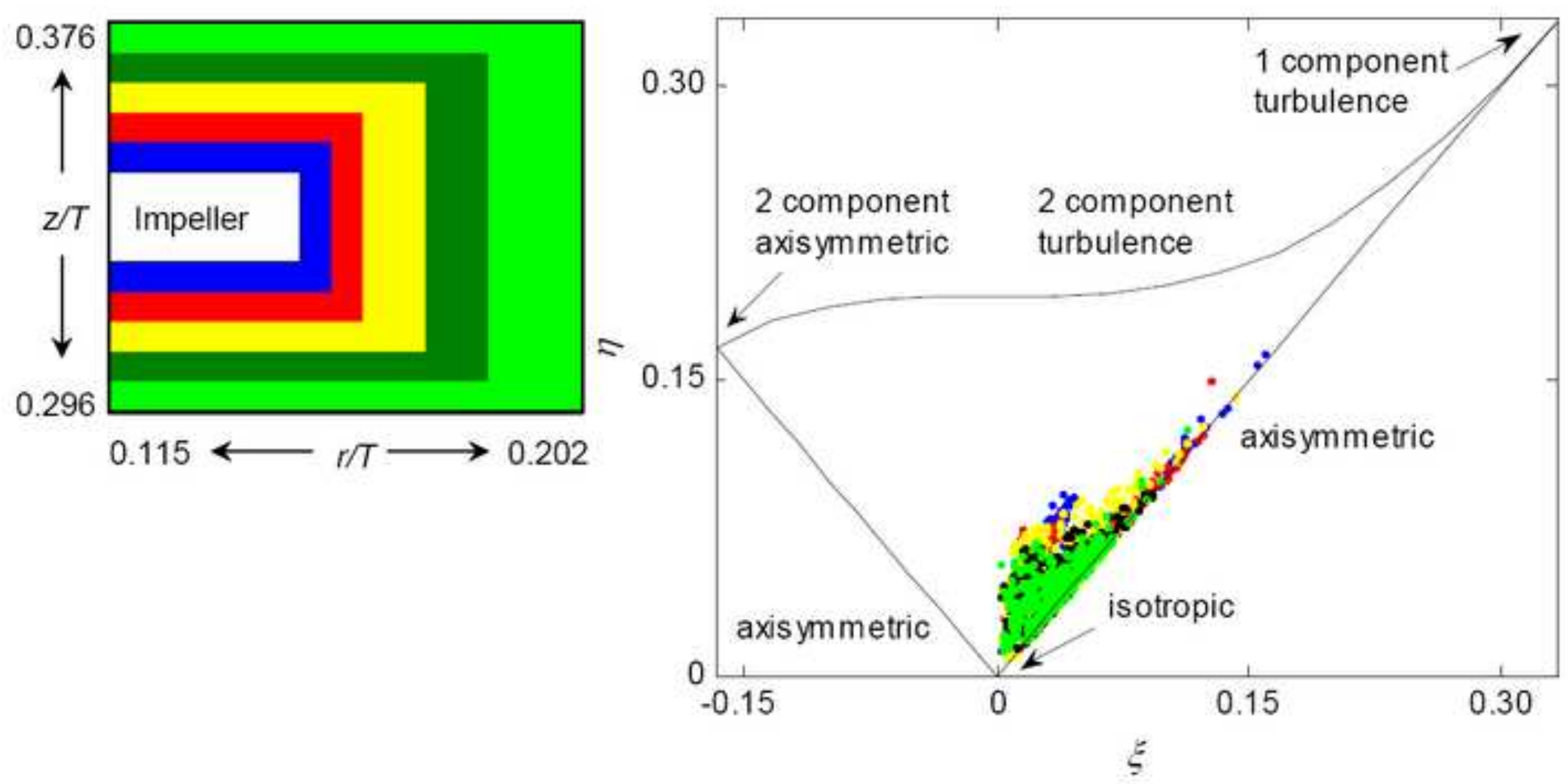


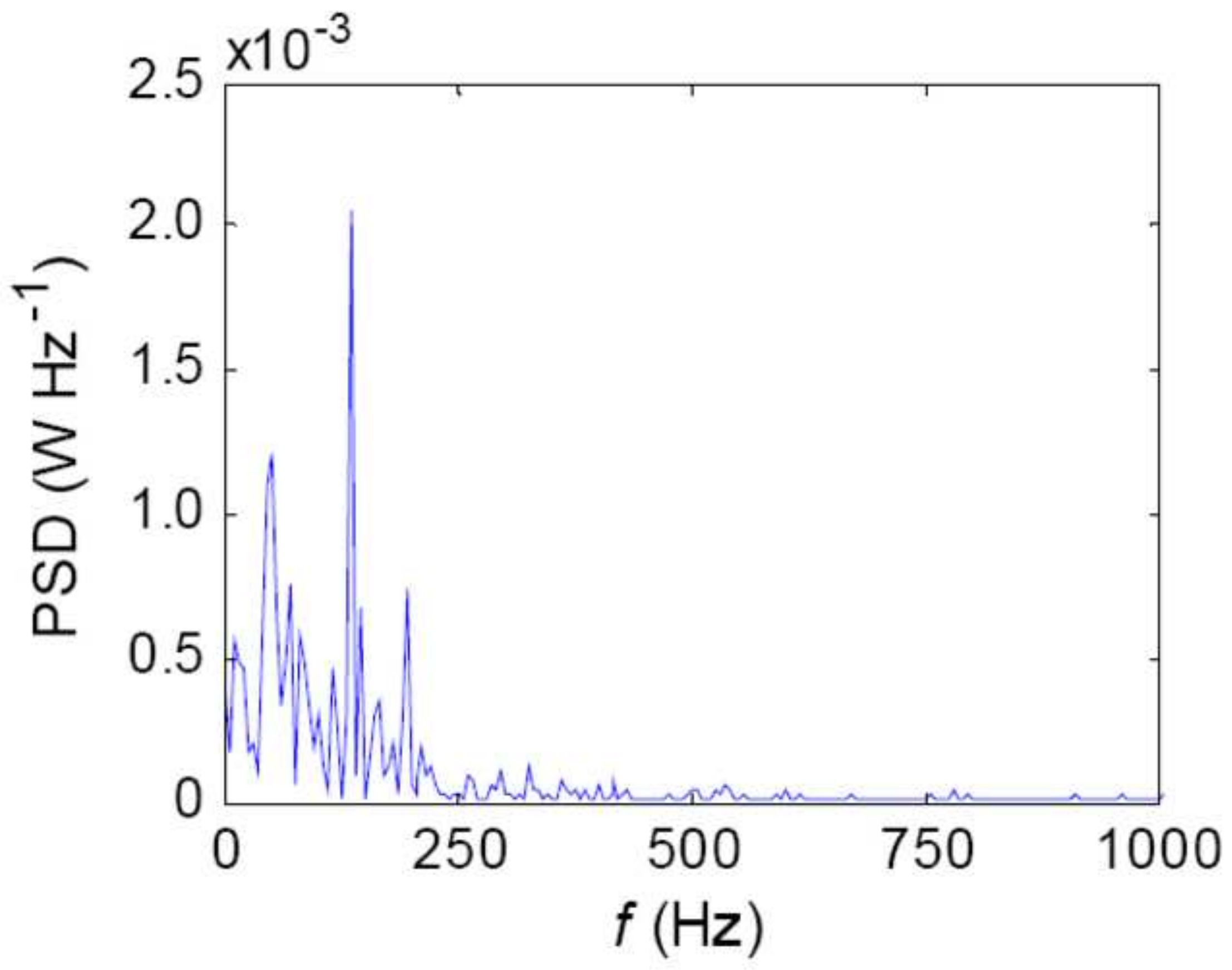




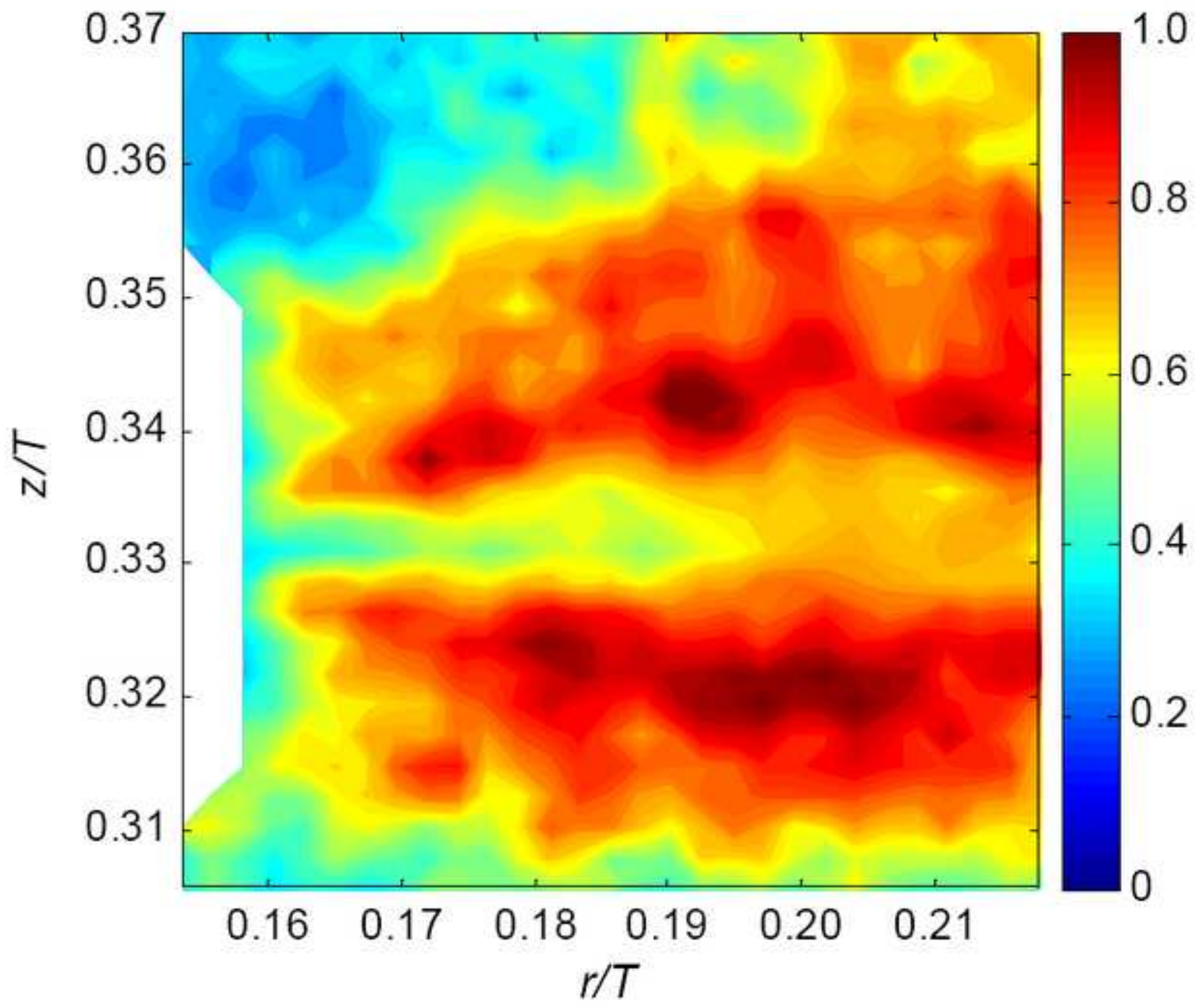




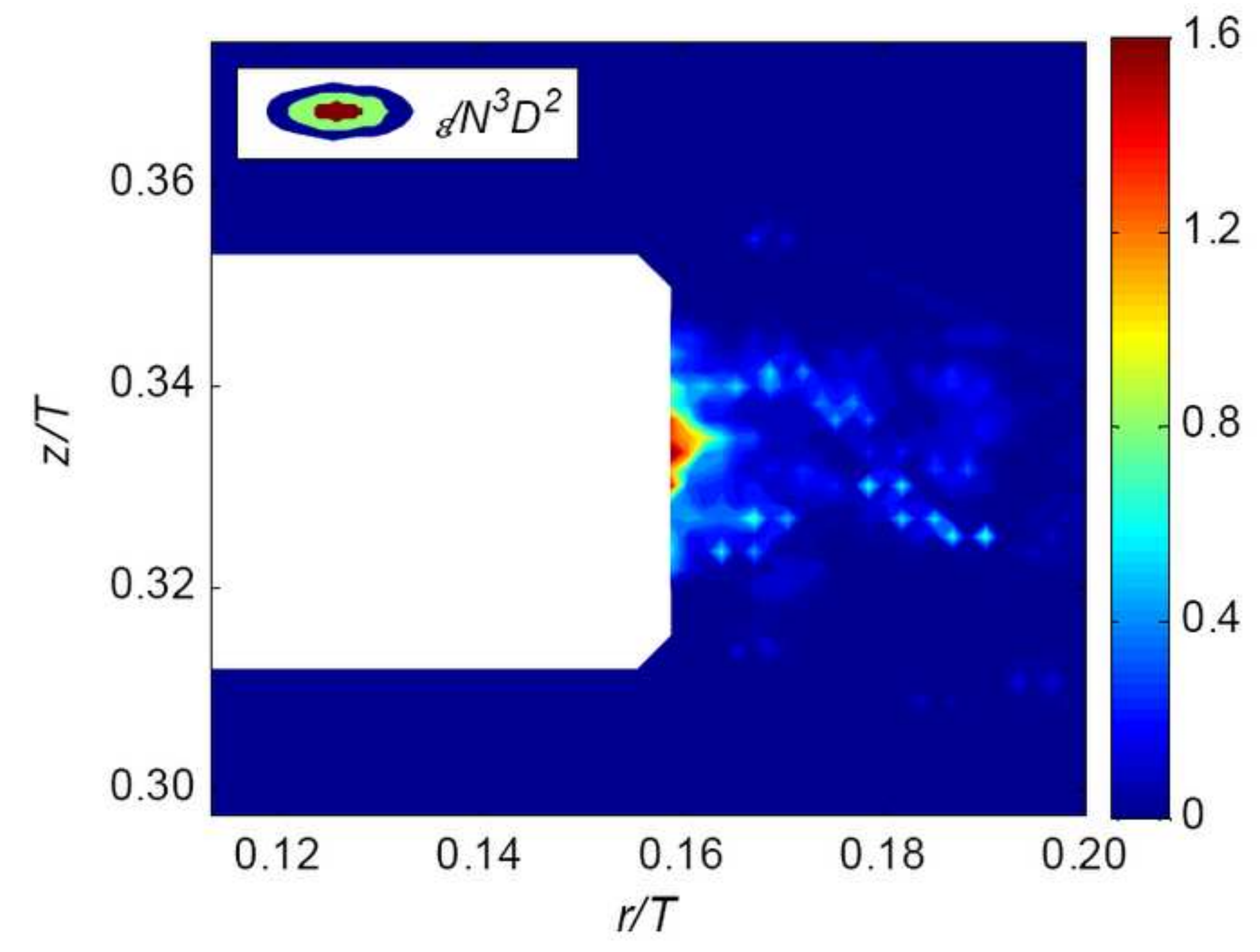




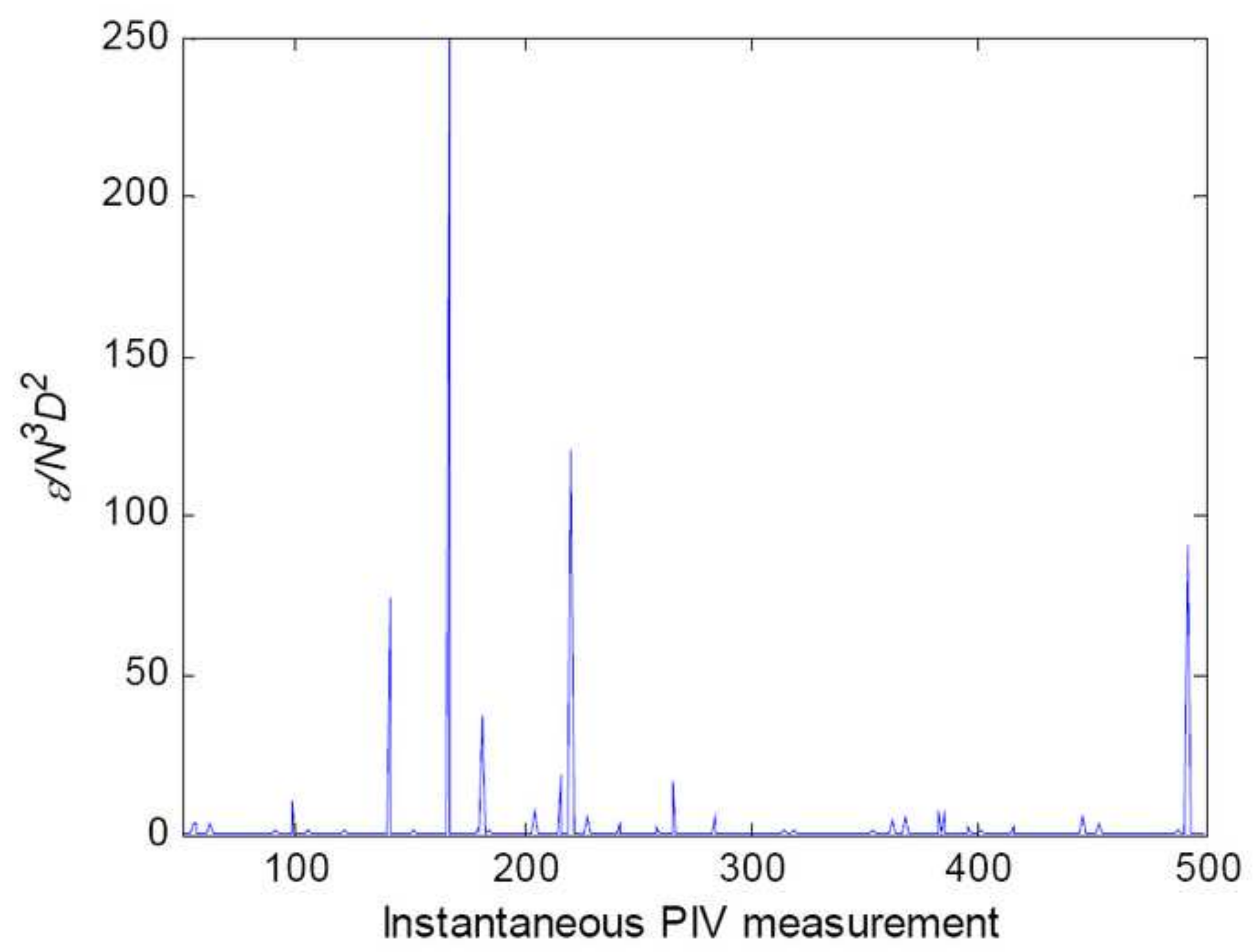




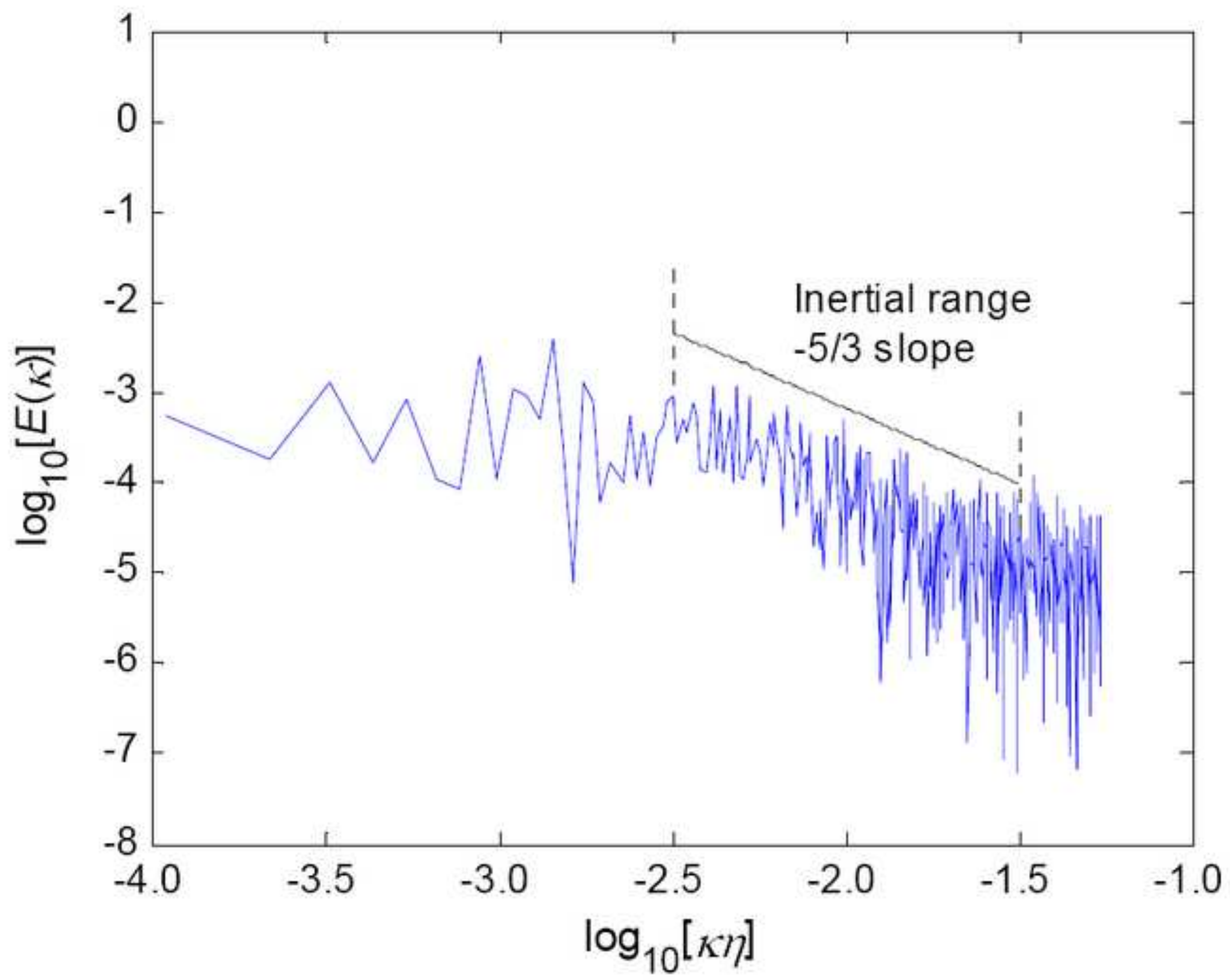




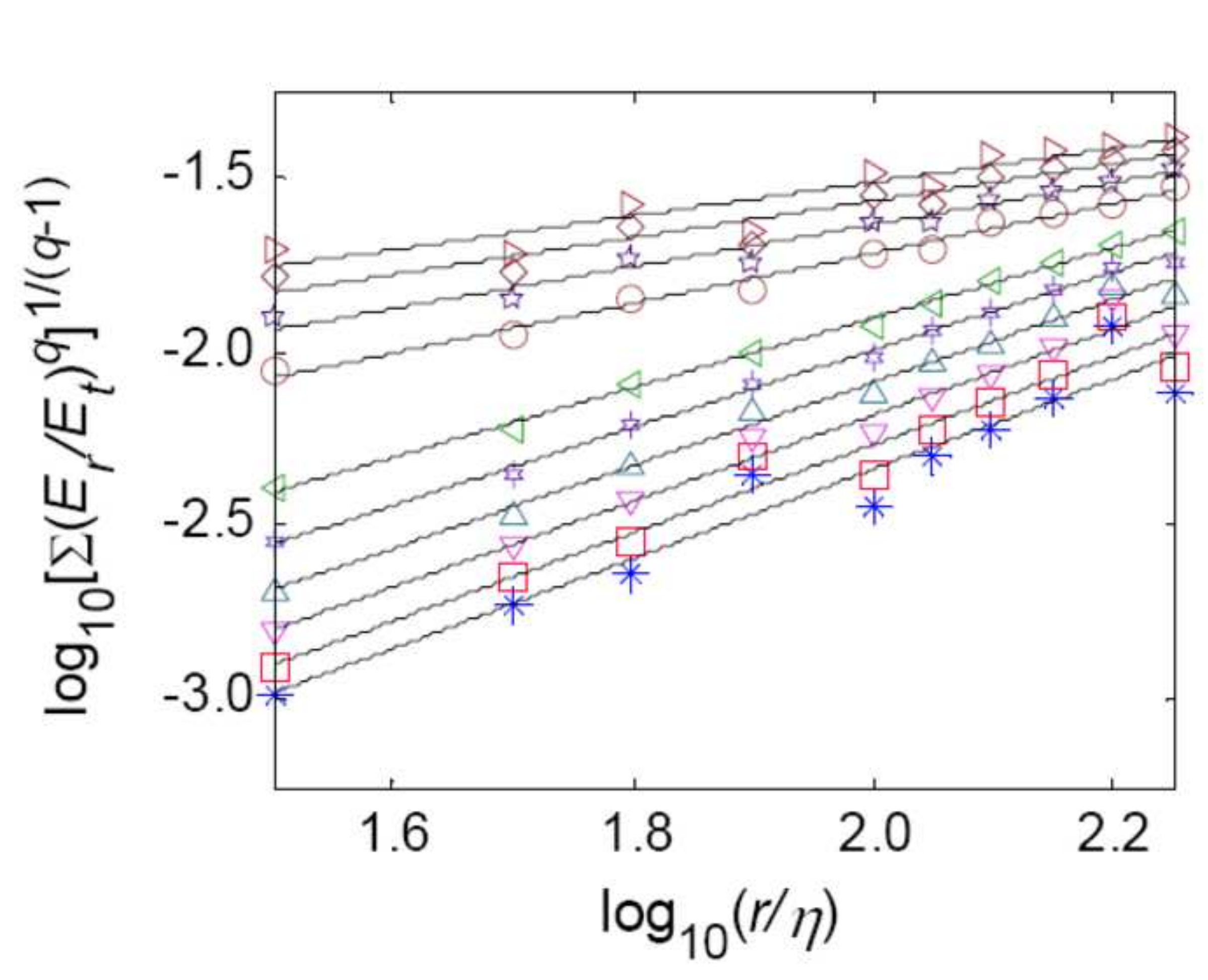




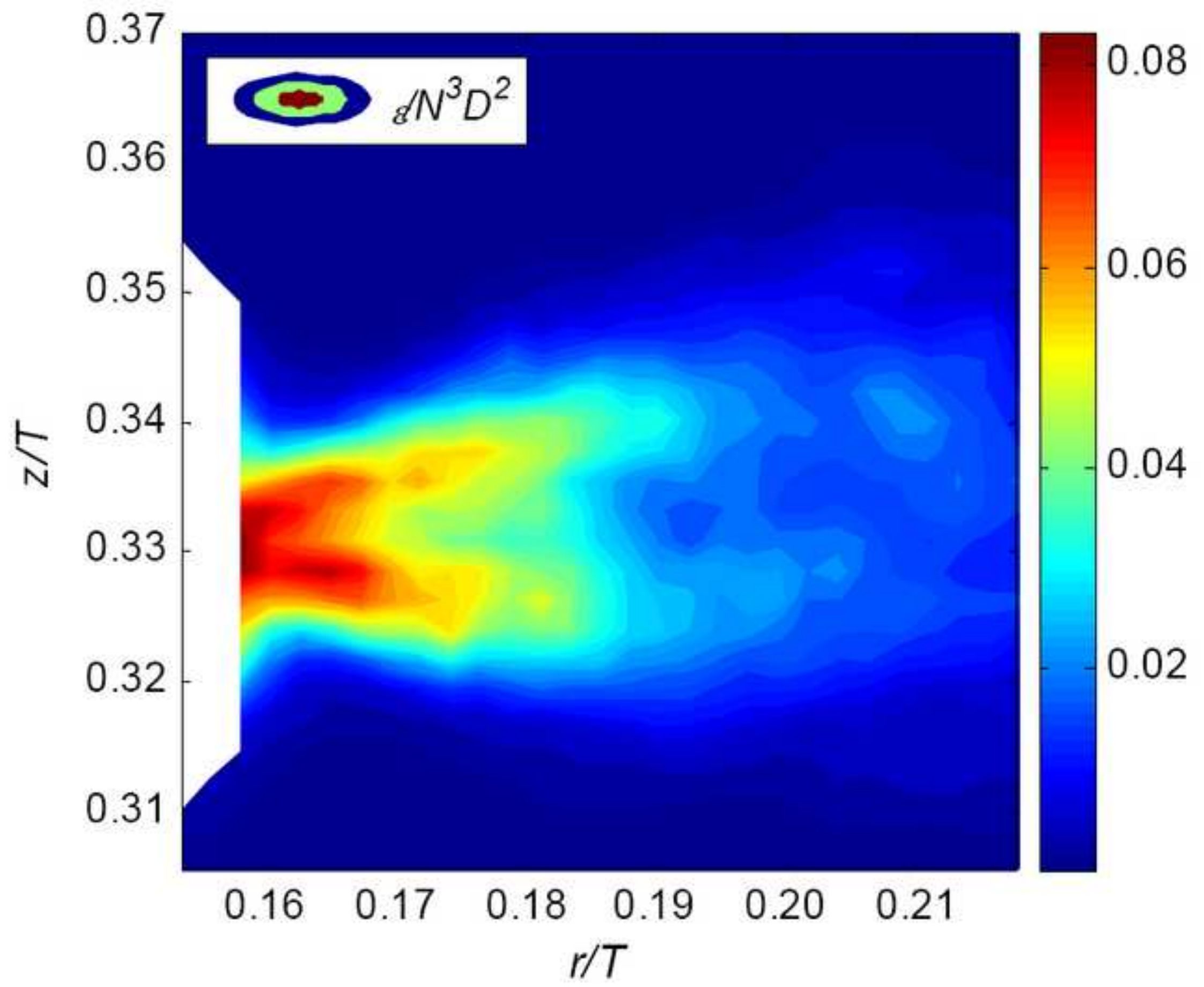




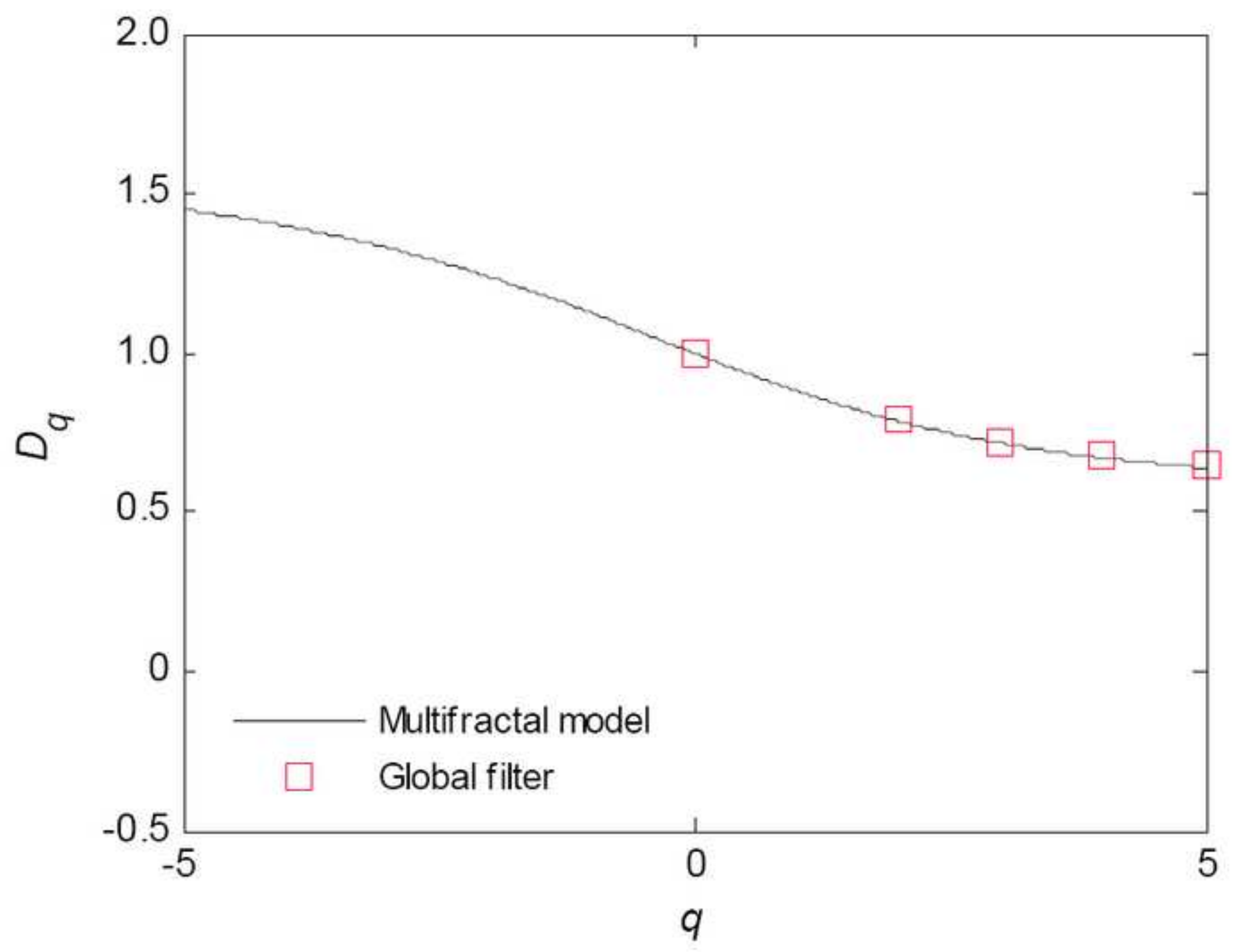




\begin{tabular}{|c|c|c|c|c|c|c|c|}
\hline Author & Impeller & $D / T$ & $P_{0}$ & $\bar{\varepsilon} / N^{3} D^{2}$ & Calc method for $\varepsilon$ & $\varepsilon_{\max } / N^{3} D^{2}$ & $\varepsilon_{\max } / \bar{\varepsilon}$ \\
\hline $\begin{array}{l}\text { Current study } \\
\text { (2009) }\end{array}$ & Sawtooth & 0.311 & 0.11 & 0.004 & $\begin{array}{l}\text { 3-D LES analogy of } \\
\text { Sheng et al. (2000) }\end{array}$ & $0.17^{* * *}$ & 40 \\
\hline $\begin{array}{c}\text { Baldi and } \\
\text { Yianneskis } \\
\text { (2003) }\end{array}$ & \multirow{2}{*}{ Hydrofoil } & 0.333 & 0.22 & 0.01 & $\begin{array}{l}\text { Directly from } \\
\text { definition }\end{array}$ & $0.02-0.8^{*}$ & $2-80$ \\
\hline $\begin{array}{c}\text { Kilander and } \\
\text { Rasmuson } \\
\text { (2005) }\end{array}$ & & 0.330 & 0.3 & 0.016 & $\begin{array}{c}\text { Modified 2-D LES } \\
\text { analogy }\end{array}$ & 14.57 & 921 \\
\hline $\begin{array}{c}\text { Zhou and } \\
\text { Kresta (1996) }\end{array}$ & \multirow{3}{*}{ PBT-4 45 } & 0.333 & 1.35 & - & $\begin{array}{c}\text { Dimensional } \\
\text { analysis }\end{array}$ & $2.44^{\star *}$ & 38 \\
\hline $\begin{array}{c}\text { Sheng et al. } \\
\text { (2000) }\end{array}$ & & 0.349 & 1.4 & 0.071 & $\begin{array}{l}\text { 2-D LES analogy of } \\
\text { Sheng et al. (2000) }\end{array}$ & 0.58 & 8 \\
\hline Khan (2005) & & 0.333 & 1.52 & 0.078 & $\begin{array}{l}\text { 2-D LES analogy of } \\
\text { Sheng et al. (2000) }\end{array}$ & 9.2 & 118 \\
\hline $\begin{array}{c}\text { Zhou and } \\
\text { Kresta (1996) }\end{array}$ & \multirow{4}{*}{ RDT-6 } & 0.333 & 5.4 & - & $\begin{array}{l}\text { Dimensional } \\
\text { analysis }\end{array}$ & $12.14^{\star *}$ & 48 \\
\hline $\begin{array}{c}\text { Sharp and } \\
\text { Adrian (2001) }\end{array}$ & & 0.333 & 4.5 & 0.209 & $\begin{array}{l}\text { Directly from } \\
\text { definition }\end{array}$ & 3.35 & 16 \\
\hline $\begin{array}{l}\text { Escudie and } \\
\text { Line (2003) }\end{array}$ & & 0.333 & 5.5 & 0.313 & TKE balance & 14 & 45 \\
\hline $\begin{array}{c}\text { Baldi and } \\
\text { Yianneskis } \\
\text { (2004) }\end{array}$ & & 0.333 & $\begin{array}{c}4 \\
4.1 \\
4.7\end{array}$ & $\begin{array}{l}0.189 \\
0.193 \\
0.222\end{array}$ & $\begin{array}{l}\text { Directly from } \\
\text { definition }\end{array}$ & $\begin{array}{l}10 \\
11 \\
6.4\end{array}$ & $\begin{array}{l}53 \\
57 \\
29\end{array}$ \\
\hline
\end{tabular}

${ }^{*}$ From PIV measurements at varying spatial resolution

${ }^{* *}$ Average $\varepsilon_{\text {max }}$ for 5 impeller speeds ***at $1500 \mathrm{rpm}$

Table 1 Maximum and mean dissipation rates of various impellers reported in literature 